\title{
Collision of a DNA Polymer with a Small Obstacle
}

\author{
Greg C. Randall and Patrick S. Doyle* \\ Department of Chemical Engineering, Massachusetts Institute of Technology, \\ Cambridge, Massachusetts 02139
}

Received June 20, 2006; Revised Manuscript Received August 1, 2006

\begin{abstract}
Using single molecule fluorescence microscopy, we study the dynamics of an electric-field-driven DNA molecule colliding with a single stationary post. The radius of the obstacle is small compared to the contour length of the molecules. Molecules that achieve hooked configurations which span the obstacle were chosen for study. Four different types of hooked configurations were found: symmetric hairpins with constant extension during unhooking, asymmetric hairpins with constant extension during unhooking, asymmetric hairpins with increasing extension during unhooking, and rare multiply looped entangled configurations. The important physics describing the unhooking dynamics for each classification differ and models are proposed to predict unhooking times. Surprisingly, we find that most collisions do not follow classic rope-on-pulley motion but instead form hairpins with increasing total extension during the unhooking process (called $\mathrm{X}$ collisions). Last, we show that unraveling to form a hairpin and center-of-mass motion during unhooking affect the overall center of mass holdup time during a collision process.
\end{abstract}

\section{Introduction}

The collision of a field-driven polymer molecule with a stationary obstacle is a simply posed but nontrivial problem. Although analogous to a classical rope-and-pulley problem, this microscale problem is complicated by the polymer's elasticity and fluctuating configuration. Collision studies have been pursued to investigate polymer-polymer interactions ${ }^{1,2}$ and the size separation ${ }^{3}$ of large DNA molecules (large DNA molecules in aqueous solvents are generally modeled using classical polymer physics ${ }^{4-6}$ ). For sufficiently strong impacts, a coiled polymer hooks around the obstacle and unwinds into a hairpin configuration. ${ }^{7}$ A hooked molecule then unhooks from the obstacle and recoils (Figure 1a). Previously, hooking dynamics of DNA in gels was hypothesized to explain anomalous experimental gel electrophoresis results ${ }^{8-11}$ and then directly observed by Song and Maestre. ${ }^{7}$ Additionally, a DNA sizeseparation design of sparse microfabricated obstacles was hypothesized by Austin and co-workers ${ }^{12-14}$ from observations of hook formation and rope-on-pulley modeling of the unhooking time.

Using simulations, other groups tested this separation hypothesis to determine length-dependent behavior of DNA mobility through a group of pointlike obstacles. ${ }^{15-20}$ These studies began by looking at the single obstacle collision event of a Rouse-like bead-spring chain at high fields. Geometric arguments were then used to scale these single collision results to an obstacle course separation device. ${ }^{17}$ Other innovations in single obstacle simulation work include study of the hooking probability on a point obstacle as a function of impact offset, ${ }^{17}$ inclusion of approximate hydrodynamic interactions, ${ }^{18}$ and introduction of the notion of "roll-off" collisions on finite size obstacles. ${ }^{19}$ Additionally, Patel and Shaqfeh ${ }^{20}$ simulated chains moving through arrays of multiple obstacles and investigated the effect of obstacle ordering and density.

The first experimental work to separate $O[10-100 \mathrm{kbp}]$ DNA by length using hooking collisions was by Doyle et al. ${ }^{3}$ Follow-

* Corresponding author: Ph (617) 253-4534, fax (617) 258-5042, e-mail: pdoyle@mit.edu.
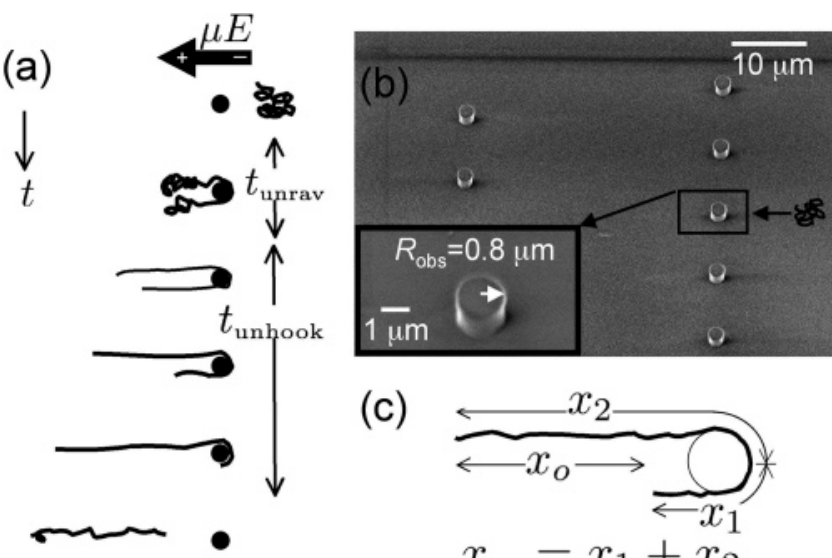

(c)

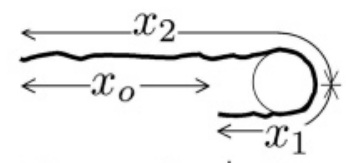

$x_{\mathrm{ex}}=x_{1}+x_{2}$

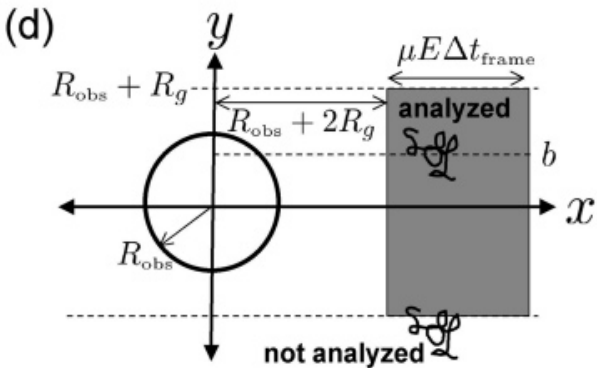

Figure 1. Single obstacle hooking of DNA. (a) Schematic of a typical hooking event. $t_{\text {unrav }}$ is the time it takes the DNA to unravel after impact. $t_{\text {unhook }}$ is the time it takes a completely unraveled DNA hairpin configuration to unhook from the obstacle. As we detail in the text, unraveling ends and unhooking begins when the short arm reaches its maximum extension. (b) SEM image of our PDMS obstacle array and an expanded view of a single post. (c) Length definitions of an unhooking DNA. $x_{1}$ is the short arm length, and $x_{2}$ is the long arm length; $x_{0}$ is the difference in arm lengths, and $x_{\mathrm{ex}}$ is the total extension of the chain. (d) Region (shaded) where DNA molecules are chosen for the impact ensemble (not to scale). Note that a collision zone a distance $2 R_{\text {obs }}+R_{\mathrm{g}}$ from the obstacle center may be a better choice, ${ }^{28}$ however, the difference is unimportant here because $R_{\mathrm{obs}} \sim R_{\mathrm{g}}$.

up work was performed in slightly more dense obstacle courses at higher fields ${ }^{21}$ and in extremely dense courses, ${ }^{22}$ though as 
the obstacle density increases, the separation mode switches from unhooking to pore sieving characteristic of gels. Motivated by these experiments, macroscale separation models were proposed to explain the dependence of the average mobility and dispersivity in an obstacle array on the DNA length. Dorfman and Viovy ${ }^{23}$ first presented a Markovian model, and then Minc, Viovy, and Dorfman adjusted the model to include nonMarkovian transport through the post array. ${ }^{24}$ To make quantitative predictions, they obtained data from a single obstacle in an array to support their claim that the unhooking time probability distribution could be modeled as a Poisson distribution. ${ }^{25}$ They argue that a better trapping model may improve agreement with experimental observations. However, no one has performed controlled microscopic experiments to extract the hold-up times for hooked DNA, and no one has constructed a comprehensive model that can predict the hold-up times for the multitude of hooking dynamics observed experimentally.

Our group attempted the first systematic experimental analysis of the collision problem by tracking a uniform ensemble of DNA molecules distributed perpendicular to the field direction impacting a small but finite size obstacle. ${ }^{26}$ Using a logical but empirical definition that a "hooking collision" was any DNA that has, at some instant, a piece of its configuration in all four quadrants of the obstacle-centered coordinate system, we extracted the hooking probability of $\lambda$ DNA ensembles on 0.8 $\mu \mathrm{m}$ radius PDMS obstacles. We found that for a small but finite size obstacle induced electric field gradients can greatly affect the hooking probability. In addition to the size ratio $R_{\mathrm{obs}} / R_{\mathrm{g}}$, the roll-off to hook transition is governed by a dynamic parameter De $=\dot{\epsilon}^{\mathrm{EL}} \tau$, the ratio of the rate of maximum DNA deformation to DNA relaxation in an obstacle-induced field gradient. It is well-known that hooking events result in order of magnitude longer and size-dependent hold-up times compared to roll-offs, ${ }^{19}$ and here we will focus solely on hooking events. In this paper we will experimentally investigate and model the hold-up time for DNA hooking on small obstacles (Figure 1a). Using fluorescence microscopy, we will experimentally extract center-of-mass hold-up times for single DNA molecules colliding with stationary obstacles. These hold-up times can be modeled by two sequential processes: unraveling and unhooking $^{16}$ (Figure 1a). Unraveling occurs after the DNA impacts the obstacle as its conformation unravels into hairpin-like configurations. Once unraveled, the DNA unhooks, as the longer arm pulls the shorter arm off of the obstacle.

We investigate the unhooking dynamics in detail, decomposing the conformations of hooked collisions into idealized ropelike collisions and nonideal variable extension collisions. Simple rope-on-pulley models have been used in the past to predict high field unhooking times of ideal ropelike collisions. ${ }^{7}$ However, when considering this entire impacting ensemble, there are many DNA that do not completely unravel, and consequently, they do not behave like a rope-on-pulley. Their existence has been observed in single obstacle simulations ${ }^{16}$ but never explored. We present collision models that can predict unhooking times for both ropelike and variable extension collisions. We also look at the unraveling mechanism for the observed collision classes. Though unhooking can generally dominate the overall hold-up time for a collision event, we will show that the unraveling process can be important to consider. Furthermore, this is the first study to investigate the importance of the center-of-mass motion on hold-up time modeling, during both unraveling and unhooking. Our work improves on previous experimental work , $^{72,27,25}$ because we examine uniform impact ensembles (DNA distributed uniformly across the width of the channel before impact), use precise center-of-mass tracking algorithms, and include all hooking events, not just visually obvious hooks.

\section{Experimental and Analytical Procedures}

Dimensionless Field Strength. First, we introduce the appropriate dimensionless field strength. The Peclet number Pe is a measure of how the persistence length of tethered DNA orients in an electric field, and it is the proper way to nondimensionalize the extension of a tethered polymer in a uniform flow $U$ or field $E$. For example, in flow, the drag force on the chain is $\xi U$ (where $\xi$ is the DNA drag coefficient), and in the low-force limit $\left(f \sim k T x_{\mathrm{ex}} /\left(L l_{\mathrm{p}}\right)\right.$, with persistence length $l_{\mathrm{p}}$ and thermal energy $\left.k T\right)$, the fractional extension $x_{\text {ex }} / L$ scales as

$$
\begin{gathered}
\frac{x_{\mathrm{ex}}}{L} \sim \frac{l_{\mathrm{p}}}{k T} U \zeta \\
=\frac{U l_{\mathrm{p}}}{D}
\end{gathered}
$$

where $D=k T / \zeta$ is the DNA's diffusion coefficient. Using electrohydrodynamic equivalence, ${ }^{29}$ we define $\mathrm{Pe}$ as $\mathrm{Pe}=\mu E l_{\mathrm{p}} / D$ in electrophoretic fields. To keep the low-force fractional extension constant, Pe must be kept constant. However, since polymer unhooking involves a competition between stretched polymer arms, it is also natural to think of $\mathrm{Pe}$ as the proper way to investigate unhooking dynamics. For Pe $>1$, a DNA that collides with a small obstacle will then stretch into a hairpin hooked configuration. Alternate versions of this dimensionless field strength have been used in the past DNA-obstacle work reviewed above. Note that Pe scales as $N^{\gamma}(v<\gamma<1$ depending on the hydrodynamic interaction screening, ${ }^{4}$ with $v=0.589$ ), which is approximately the length scaling Perkins et al..$^{30}$ empirically determined to achieve a family of universal extension curves of tethered DNA. Additionally, the free draining version $(\gamma=1)$ of Pe was employed by Patel et al. ${ }^{20}$ to collapse a family of Rouse bead-spring chain unhooking simulations onto one master collision time curve. Generally Pe $O[10]$ in recent DNA separation devices. ${ }^{3,21}$

We stress that the governing dimensionless field strength $(\mathrm{Pe})$ is very different from the Deborah number (De) used in our previous paper that examined the transition between hooks and roll-offs. ${ }^{26}$ De governs deformation of polymers in field gradients, e.g., the gradients induced by an insulating obstacle, and thus is useful when studying the DNA deforming around the obstacle. Because most of the hooked DNA studied here stretch into regions where the field is uniform, the electric field gradients induced by the obstacles are of secondary importance in the unraveling/unhooking analysis.

Experimental Procedure. We used a standard soft lithography procedure (described elsewhere) ${ }^{31,32}$ to construct $25 \mathrm{~mm}$ long, 50 $\mu \mathrm{m}$ wide, and $2 \mu \mathrm{m}$ high PDMS (poly(dimethylsiloxane)) microchannels with a sparse array of $0.8 \mu \mathrm{m}$ radius obstacles. The obstacles were spaced $70 \mu \mathrm{m}$ center-to-center in the field direction and were staggered transverse to the field with a $10 \mu \mathrm{m}$ center-tocenter spacing. Reservoirs $(4 \mathrm{~mm} \times 4 \mathrm{~mm})$ were cut at each end of the cured PDMS microchannel with a scalpel, and the channels were soaked for $12 \mathrm{~h}$ at $45^{\circ} \mathrm{C}$ in $0.5 \times \mathrm{TBE}$ to eliminate permeation driven flow. ${ }^{32}$ Figure $1 \mathrm{~b}$ shows a SEM image of the obstacle array and an isolated obstacle.

We used $\lambda$ DNA and T4 DNA in this study. The DNA were stained with a fluorescent dye (TOTO-1, 4.7:1 bp:dye molecule) and diluted in the following buffers: (1) $2.2 \times \mathrm{TBE}, 3 \%$ $\beta$-mercaptoethanol, $0.07 \%$ PVP $\left(M_{\mathrm{w}}=10^{6}\right)$, and $0.07 \%$ ascorbic acid (used for $\lambda$ DNA) or (2) $2.2 \times$ TBE, $3 \% \beta$-mercaptoethanol, and $0.07 \%$ PVP $\left(M_{\mathrm{w}}=10^{4}\right)$ (used for T4 DNA). The additives were chosen to dynamically eliminate electroosmotic flow and scavenge oxygen. The measured electrophoretic mobility was $\mu=$ $-1.7 \pm 0.2(\mu \mathrm{m} / \mathrm{s}) /(\mathrm{V} / \mathrm{cm})$. As explained previously ${ }^{28}$ the dyed $\lambda$ 
DNA has a contour length of $L=21 \mu \mathrm{m}$ and a longest relaxation time of $\tau=0.19 \mathrm{~s}$. Using the same procedures, we determined T4 has a stained contour length of $70 \mu \mathrm{m}$ and $\tau=1.7 \mathrm{~s}$.

A typical experiment consisted of first gently rinsing and drying the microchannel and then applying it to a clean glass slide (plasma cleaned at $100 \mathrm{~W}$ for $5 \mathrm{~min}$, charge equilibrated 1 day, soaked in $1 \mathrm{M} \mathrm{NaOH}$ for $15 \mathrm{~min}$, and rinsed in ultrapure water (Milli-Q, Millipore)). We then immediately filled the channel with DNA solution and applied an electric field of $5.2-23.4 \mathrm{~V} / \mathrm{cm}$ across the reservoirs through platinum electrodes. We observed single DNA molecule dynamics using an inverted fluorescence microscope (Axiovert 200, Zeiss) with a $100 \times 1.4$ NA objective for $\lambda$ DNA and a $63 \times 1.4$ NA objective for T4 DNA. Images were captured at $30 \mathrm{frames} / \mathrm{s}\left(\Delta t_{\text {frame }}=0.0333 \mathrm{~s}\right)$ with an EB-CCD camera (C7190-20, Hamamatsu) and NIH Image software. Digitized images had 8 bit pixel intensity values which ranged from 0 to 255 .

Analysis. To extract data, we first filtered the background noise by subtracting the maximum pixel value of the perimeter of the first frame of a movie from all the pixels in that movie. The primary observable from the captured images of a DNA collision was the center-of-mass coordinate of the DNA $\left(x_{\mathrm{com}}, y_{\mathrm{com}}\right)$. We adopted an obstacle-centered coordinate system for each collision, with the $x$-coordinate in the field direction and the $y$-coordinate transverse to the field (Figure 1d). The center of mass of a molecule was computed by finding the first moment of the postfiltered image intensity distribution. ${ }^{33}$ This process was automated for multiple frames of data, and it yielded the center-of-mass trajectory $\mathbf{x}_{\mathrm{com}}(t)$ of the DNA. We also determined the extension of the short $\left(x_{1}\right)$ and long $\left(x_{2}\right)$ arms of an unhooking DNA by the distance between the end of each arm and the center of the obstacle plus a geometric term $\pi R_{\mathrm{obs}} / 2$ (Figure 1c). The total extension $x_{\mathrm{ex}}$ of the DNA is then $x_{\mathrm{ex}}=x_{1}+x_{2}$. Furthermore, we define the difference in arm lengths $x_{0}$ as $x_{0}=x_{2}-x_{1}$. Refer to Figure 1c for a diagram of these observable lengths.

We obtain obstacle positions for each 2000 frame movie of data by averaging the pixel values of all frames. Obstacles are easily observed as vacancies in the smeared DNA traces of the pixel average. We then define the impact parameter $b$ for a collision as the $y$-offset between the impacting DNA's center of mass and the obstacle center, taken at a distance $R_{\mathrm{obs}}+2 R_{\mathrm{g}}$ from the obstacle center (Figure 1d). This position was chosen to evaluate $b$ because the DNA is far enough away from the obstacle to not experience high obstacle-induced electric field gradients, yet close enough so that Brownian drift does not significantly coarsen our analysis. For this study, we only analyze collisions with $|b|<R_{\mathrm{obs}}+R_{\mathrm{g}}$. No hooking collisions were observed for DNA with $|b|>R_{\mathrm{obs}}+R_{\mathrm{g}}$.

Using the center-of-mass trajectories of the colliding DNA, we can compute the collision hold-up time $t_{\mathrm{H}}$. For all collisions we consider, the coiled DNA is moving from right to left, i.e., from positive $x$ to negative $x$. A typical $x_{\text {com }}(t)$ trajectory (T4 DNA, Pe $=8$ ) is shown in Figure 2. A typical collision shows a decrease in $x_{\text {com }}(t)$ until the DNA impacts the obstacle $(x=0)$. Impact is then followed by a plateau in $x_{\text {com }}(t)$ when the molecule is hooked on the obstacle. At this point the DNA configuration resembles a hairpin (Figure 2, inset). Finally, when the molecule unhooks, $x_{\text {com }}(t)$ decreases again. The pre- and postimpact slope of $x_{\text {com }}(t)$ should be equal to $\mu E .^{52}$ Within $10 \%$ experimental error, all molecules move at $\mu E$ both before and after colliding with the obstacle. We extract $t_{\mathrm{H}}$ for each collision as the difference in time intercepts for lines fit to the approach and release trajectories (Figure 2). The linear fit for the approach data uses two fit parameters (slope and intercept), whereas the linear fit for the release data uses just an intercept because the slope is constrained to match the approach slope. The approach data is fit from the first data point until $x_{\mathrm{com}}$ $<R_{\mathrm{obs}}+2 R_{\mathrm{g}}$. The release data is fit from when $x_{\mathrm{com}}<-x_{\mathrm{ex}, \max } / 2$, where $x_{\text {ex,max }}$ is the maximum total extension of any DNA in a given ensemble. One of the primary goals of this paper is to compare experimental results for $t_{\mathrm{H}}$ to collision models that incorporate effects from unhooking and unraveling.

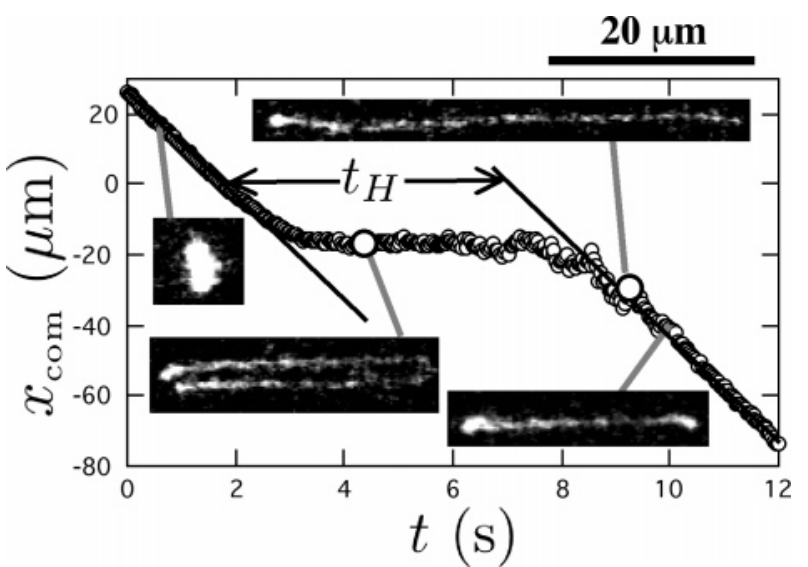

Figure 2. A sample collision trajectory ( $\mathrm{T} 4$ at $\mathrm{Pe}=8$ ) along with characteristic DNA configurations. The large circle data points mark the beginning and ending of unhooking. The first large circle represents when the short arm begins to retract, and the second large circle represents when the DNA leaves the obstacle.

\section{Hooking Collision Classification}

Figure 3 shows experimental images of different T4 molecules hooking on a single obstacle at $\mathrm{Pe}=8$. We will classify hooking collisions into four general categories named for their shape: $\mathrm{U}, \mathrm{J}, \mathrm{W}$, and X (for "extending") collisions. U and J collisions have a relatively constant extension $\left(x_{\mathrm{ex}}=x_{1}+x_{2} \sim\right.$ constant $)$ during the unhooking process and behave like a rope on a frictionless pulley. This constant extension, which we term $\mathscr{L}$, depends on Pe. $U$ and $J$ collisions are the types of ideal collisions described in previous studies. ${ }^{7,12,25,27}$ The main distinction is that $U$ collisions (Figure 3a) have nearly symmetric arms so that arm length fluctuations are important to consider when modeling the unhooking time. W collisions (Figure 3c) are collisions that result in entangled configurations upon impact with the obstacle (see Figure $3 \mathrm{c}$, inset). Quite often they result from the two ends of the DNA ending up on the same side of the obstacle after impact. X collisions (Figure 3d) are those with a long arm that is still extending while the short arm begins to retract.

In this work we do not quantitatively distinguish between $\mathrm{U}$ and $\mathrm{J}$ collisions. The main qualitative distinction is the increased importance of fluctuation effects in the unhooking dynamics for $U$ collisions. We will return to this point later when discussing unhooking models. We distinguish the $\mathrm{U} / \mathrm{J}$ collisions from the $\mathrm{X}$ collisions simply based on their total extension when the short arm begins to retract; U/J collisions have $x_{\mathrm{ex}}=\mathscr{L}$ whereas $\mathrm{X}$ collisions have $x_{\mathrm{ex}}<\mathscr{L}$. We determine $\mathscr{f}$ at a given Pe by taking the average of $x_{\mathrm{ex}}$ for only hooking events that result in symmetric arms. Obviously there will be some spread in the extension data, so we empirically classify molecules with $x_{\text {ex }}$ within $10 \%$ of $\mathscr{L}$ to be a U/J collision. This is a good approximation since the distribution of $x_{\mathrm{ex}}$ at the onset if unhooking is bimodal with a minimum near 0.9 $\mathscr{L}$. Also, $\mathscr{L}$ is approximately equal to the extension derived from empirical master curves for tethered DNA at steady state in uniform flows and fields in refs 30 and 34.

Figure 4 shows plots of the lengths of the short arm, long arm, and total extension (nondimensionalized by $\mathscr{L}$ ) of $\mathrm{U}, \mathrm{J}$, and $\mathrm{X}$ collisions during the unraveling and unhooking processes; similar plots for the $U$ collision were presented in refs 7 and 15. The solid lines beginning at the left axes are affine scalings. Although we present only three examples of each collision type's arm length trajectory, we note some universal trends. During unraveling, the arms extend approximately at a velocity 


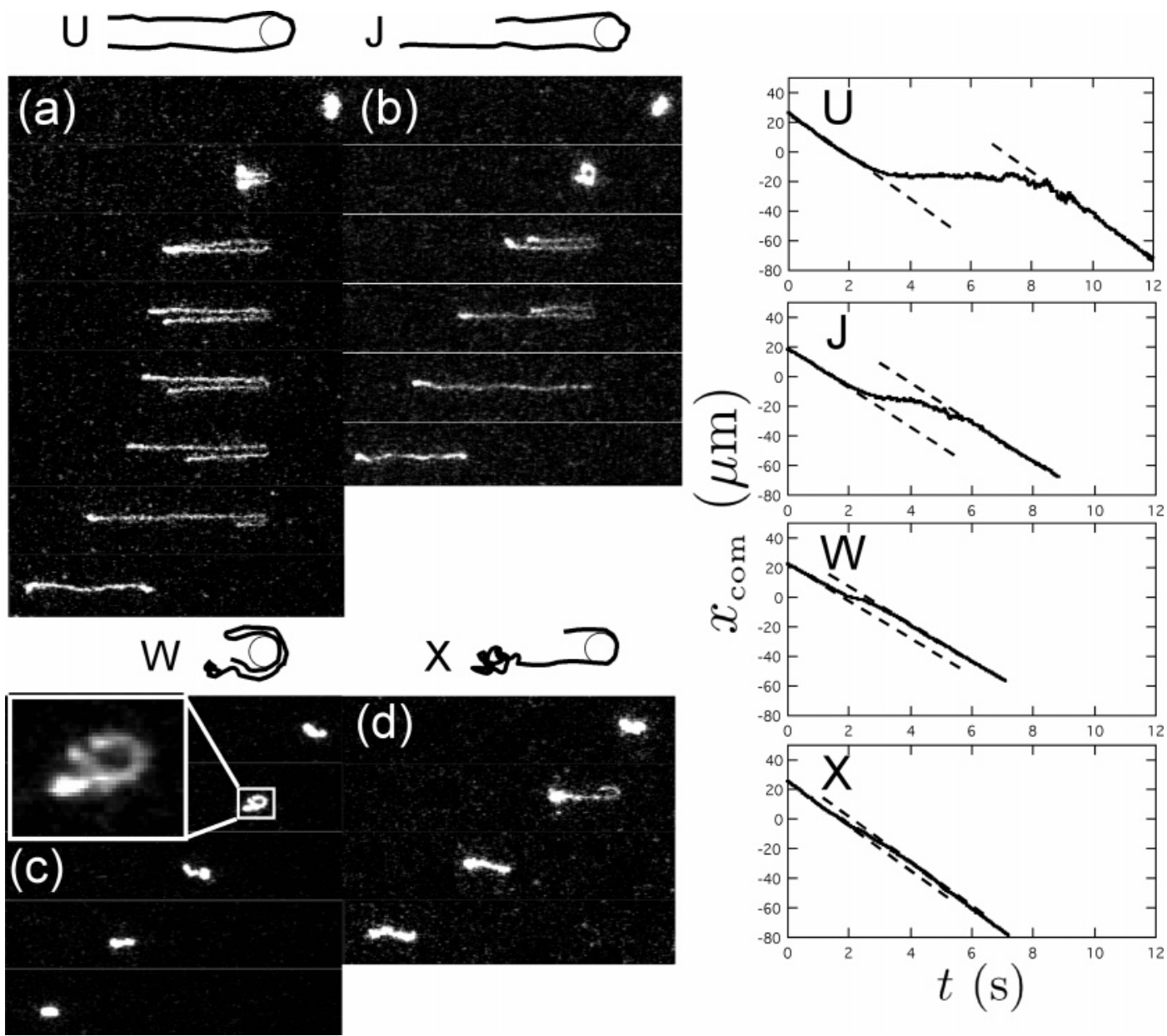

Figure 3. Types of hooking collisions (T4 DNA at Pe $=8$ ): (a) U symmetric hooks, (b) J asymmetric hooks, (c) entangled W hooks, and (d) continuously extending $\mathrm{X}$ collisions. Images in each series are separated by $1.33 \mathrm{~s}$. The plots to the right of the DNA images correspond to the $x$-component of each DNA's center-of-mass trajectory. The dashed lines show linear fits to approach and release data.

$\mu E$. Also, during unhooking (1) $\mathrm{U}$ and $\mathrm{J}$ collisions have a relatively constant total extension $\left(x_{\mathrm{ex}} / \mathscr{L} \sim 1\right)$ with exponential growth/decay of the two arms and (2) $\mathrm{X}$ collisions have $x_{\mathrm{ex}} / \mathscr{L}<1$ and $x_{\mathrm{ex}} / \mathscr{L}$ grows for the duration of the unhooking process. Furthermore, the long arm of $\mathrm{X}$ collisions tends to grow linearly, leading to the conclusion that the long arm of an X hook is still extending in the uniform field even while the short arm retracts. We will return to Figure 4 later to discuss model predictions of the arm length dynamics.

For the T4 DNA at $\mathrm{Pe}=8$ (e.g., Figure 3), 60\% of the hooking collisions were $\mathrm{X}$ collisions, $29 \%$ were U/J collisions, and $11 \%$ were $\mathrm{W}$ collisions. About half of the $\mathrm{W}$ collisions are metastable, meaning that they eventually unravel into one of the other collision classes before the molecule unhooks. The other half quickly disengage from the obstacle. Similar dynamics of multilooped hooks were hypothesized in the simulation work of refs 17 and 18 and modeled in ref 35 . Table 1 summarizes our classification results for our three main studies. Note that the number of X collisions grows with Pe. At high Pe, tensions grow nonlinearly in the short arm that can lead to quick retraction, whereas at low Pe, thermal energy has a greater effect on stabilizing asymmetric configurations. This result suggests that infinite-Pe rope-on-pulley models may not be as applicable to real polymer collisions since most collisions will be $\mathrm{X}$ collisions. The number of $\mathrm{W}$ collisions grows with size and is presumably a function of $R_{\mathrm{obs}} / R_{\mathrm{g}}$. This makes sense since at small $R_{\mathrm{obs}} / R_{\mathrm{g}}$ configuration folds are more likely to wrap around both sides of the obstacle. We note, however, that in the point obstacle limit the simulations of ref 17 appear to show only a small percentage $(<5 \%)$ of significantly observable (metastable) $\mathrm{W}$ collisions.

Like the "molecular individualism" behavior of polymers in extensional fields, ${ }^{36,37}$ we anticipate these collision types to be highly dependent on the impacting DNA's initial configuration. Similarly, classification of collision types is more powerful than a qualitative observation, as it can lead to quantitative conclusions. Specifically, the resulting hold-up times will be different for each class of collision types. The center-of-mass trajectories for each example collision in Figure 3 give a feel for this, as $U$ collisions tend to have very long hold-up times, while $\mathrm{X}$ collisions can have very short times. The total hold-up time can be thought of as a sum of the time it takes the DNA to unravel and then unhook from the obstacle. ${ }^{16}$ The unhooking time will generally be much longer than the unraveling time, so we will begin our modeling discussion there. Knowledge of how long it takes these hooked configurations to unravel and unhook can lead to useful predictive models of a macroscale separation process.

\section{Unhooking Time Models}

In this section, we develop and test mathematical models for the unhooking process. These models will only be for $\mathrm{U}, \mathrm{J}$, and $\mathrm{X}$ collisions and will not consider the more unpredictable but rare $\mathrm{W}$ collisions. Two unhooking time models will be presented: a deterministic model for constant extension chains ( $\mathrm{J}$ model) and a deterministic model for extending chains (X 


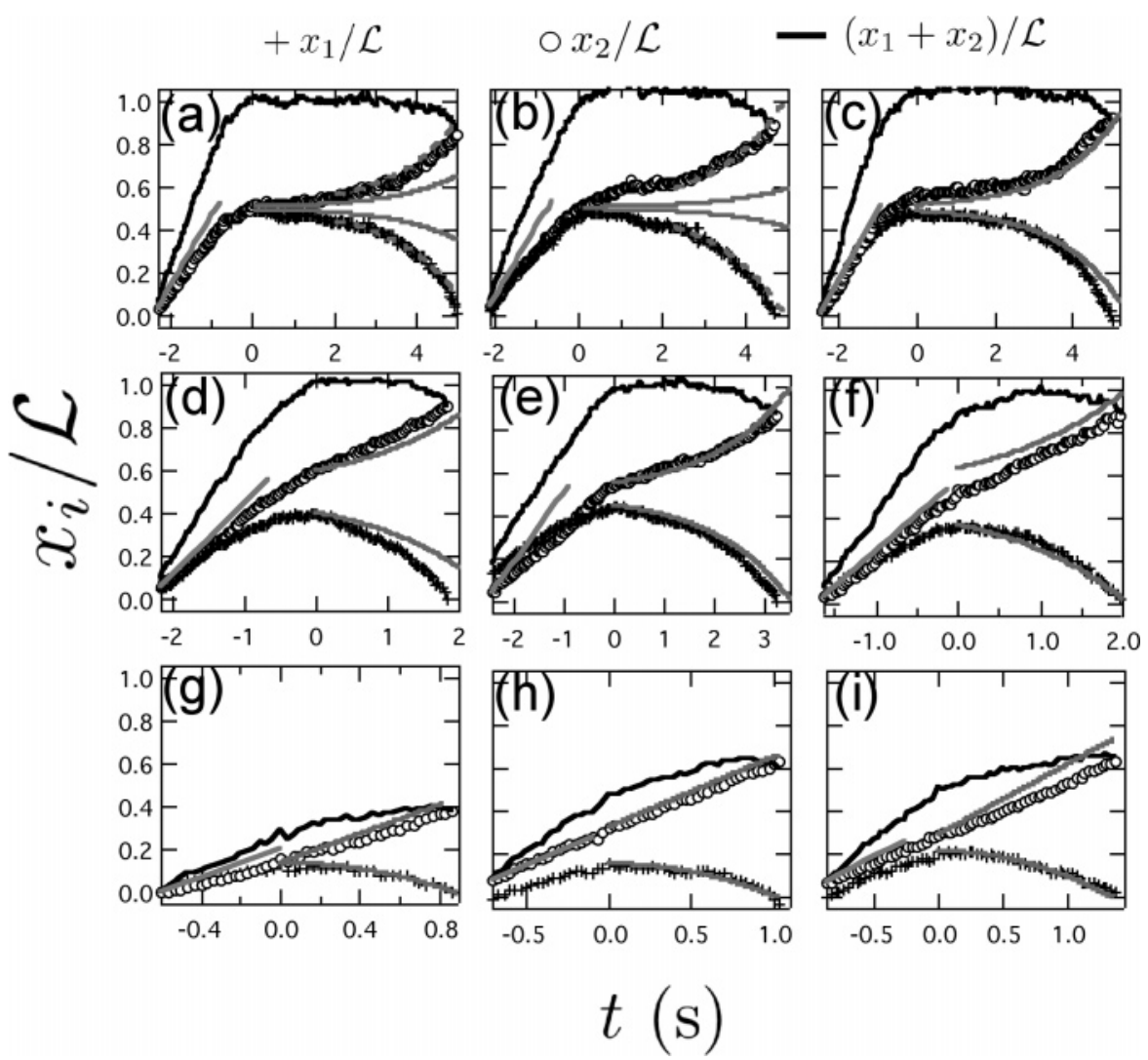

Figure 4. Arm length dynamics of (a)-(c) $\mathrm{U},(\mathrm{d})-(\mathrm{f}) \mathrm{J}$, and (g) $-(\mathrm{i}) \mathrm{X}$ hooking collisions. The trajectories are of $x_{1}(t), x_{2}(t)$, and $x_{\mathrm{ex}}(t)=x_{1}+x_{2}$ scaled by the constant $\mathrm{U} / \mathrm{J}$ extension $\mathscr{S}=45 \mu \mathrm{m}$ for T4 DNA at Pe $=8$. We normalize time to $t=0$ at the maximum value of $x_{1}(t)$, i.e. when the short arm begins to retract. (a), (d), and (g) correspond to the same DNA shown in Figure 3. Solid lines beginning at the left axis correspond to affine movement at the ensemble average electrophoretic velocity. Solid lines beginning at $t=0$ correspond to the model predictions for $x_{1}(t)$ and $x_{2}(t)$ based on initial conditions $x_{1}(0)$ and $x_{2}(0)=\mathscr{f}-x_{1}(0)((\mathrm{a})-(\mathrm{f}) \mathrm{J}$ model, (g) $-(\mathrm{i}) \mathrm{X}$ model). In (a) - (c), an arbitrary alternate initial condition was chosen at time $t$ with $x_{1}(t)<0.45$ (dashed line $\mathrm{J}$ model). The dashed line is underneath the solid line in (c).

Table 1. Summary of Hooking Collision Classification Results

\begin{tabular}{|c|c|c|c|}
\hline (DNA, Pe) & $\mathrm{U} / \mathrm{J}$ & $\mathrm{X}$ & W \\
\hline$(\lambda, \mathrm{Pe}=2)$ & $83 \%$ & $17 \%$ & $0 \%$ \\
\hline$(\lambda, \mathrm{Pe}=8)$ & $43 \%$ & $57 \%$ & $0 \%$ \\
\hline$(\mathrm{T} 4, \mathrm{Pe}=8)$ & $29 \%$ & $60 \%$ & $11 \%$ (5\% metastable) \\
\hline
\end{tabular}

model). For the remainder of this paper, we will appropriately normalize time to $t=0$ at the end of unraveling and the onset of unhooking. Empirically, this is when the short arm reaches its maximum extension. Consequently, at $t=-t_{\text {unrav }}$ the DNA is first impacting the obstacle and at $t=t_{\text {unhook }}$ the DNA is just leaving the obstacle. In the following analysis, we will use the well-accepted assumption ${ }^{14}$ that friction on the obstacle is negligible because thermal fluctuations in the solvent ensure that the DNA is not atomically pinned against the solid surface. ${ }^{38}$

Unhooking Physics. At first glance, it is clear that unhooking is driven by asymmetry in the arms; the longer arm will naturally pull the shorter arm around and off of the obstacle. To predict the unhooking dynamics, we need to know the forces at play. It is clear that the tension profiles along both arms are not at steady state because the tension would then be discontinuous at the obstacle pivot point. However, we do know that a subset of hooking collisions achieve constant extension $\mathscr{L}$. This would be true if the molecule unhooks on a time scale much shorter than the stress relaxation time, so that the tension profile in each arm can be thought of as frozen like a constant extension rope. ${ }^{15}$ As a consequence, the location of maximum tension at the start of unhooking would move onto and then down the long arm over time. This is obviously an oversimplification, and in fact we know that unhooking times are often longer than the longest stress relaxation time $\left(t_{\text {unhook }}>\tau\right)$. However this picture may capture the important physics of the chain release. In fact, the simulations of Sevick and Williams showed that the position of maximum tension does shift onto the long arm of the DNA during unhooking. ${ }^{15}$ Furthermore, even if the initial tension distribution does relax during the unhooking process, bead-rod simulations show that because of nonlinear elasticity, very large changes in tension can result from very small shifts in a stretched configuration. ${ }^{39}$ Therefore, the first-order model for an unhooking DNA is to consider an unhooking chain of constant length; we will consider both complete extension $(L)$ and moderate extension $(\mathscr{L})$. We will term a model with constant extension the "J model" since it likely best describes J collisions. The $\mathbf{J}$ model is analogous to previously proposed full extension "rope-on-pulley" models; ;,14,15 however, we will later show the benefit of formulating it in terms of the short arm length $x_{1}$.

J Model. Previously, a collision model based on rope-onpulley dynamics was used to model highly stretched DNA unhooking at high fields. ${ }^{7,14}$ The unhooking driving force is $\lambda E x_{0}$, where $\lambda$ is a phenomenological charge per length driving DNA unhooking and $x_{0}$ is the difference in contour length of the two arms. Choosing a linear driving force may seem somewhat arbitrary at first glance given that DNA and other polymers have nonlinear elasticity at full extension. Consequently, we will go through a detailed derivation of the driving force using a bead-spring picture and then more generally apply results to moderately extended chains.

Figure 5a shows a schematic of the bead-spring polymer hooked on an obstacle. First, in agreement with previously 
proposed rope-on-pulley models, we will consider a chain stretched to its full contour length so that $x_{1}+x_{2}=L$. At the small length scale of a DNA persistence length, inertia is negligible so that the forces on a small piece of the DNA sum to zero. Consequently, in the bead-spring picture of polymer dynamics, the velocity of one bead of a tethered chain in a uniform electric field without intrachain hydrodynamic interactions and without solvent-induced Brownian forces is

$$
\frac{\mathrm{d} \mathbf{x}_{n}}{\mathrm{~d} t}=\frac{1}{\zeta_{n}} \mathbf{T}\left(\mathbf{x}_{n}\right)+\mu \mathbf{E}
$$

where $\zeta_{n}$ is the bead drag coefficient, and for the $n$th bead, $\mathbf{x}_{n}$ is the position vector, $\mathbf{T}\left(\mathbf{x}_{n}\right)$ is the force due to gradients in tension along the polymer, and $\mathbf{E}$ is the imposed electric field. Ignoring Brownian forces is a good approximation for $\mathrm{Pe} \gg 1$ for asymmetric hooked configurations. Neglecting long-range bead-bead hydrodynamic interactions is a good approximation for a stretched polymer in a thin slit; ${ }^{40}$ however, our $2 \mu \mathrm{m}$ channels only partially screen hydrodynamic interactions. ${ }^{41}$ Nevertheless, including preaveraged hydrodynamic interactions would only affect our analysis parametrically through the term $\zeta_{n}$, which eventually drops from the unhooking time equations. The net spring force at bead $n$ is $\mathbf{T}\left(\mathbf{x}_{n}\right)=\mathbf{T}_{n}^{\prime}+\mathbf{T}_{n}$, where $\mathbf{T}_{n}^{\prime}$ is the force from the spring connecting bead $n$ to bead $n+1$ and $\mathbf{T}_{n}$ is the force from the spring connecting bead $n$ to bead $n-1$. Note that $\mathbf{T}_{n}^{\prime}=-\mathbf{T}_{n+1}$ except for the special case at the pivot spring $j$ where $\mathbf{T}_{j}^{\prime}=\mathbf{T}_{j+1}$. Also note that $\mathbf{T}_{1}=\mathbf{T}_{N_{\mathrm{b}}}^{\prime}=0$, where $N_{\mathrm{b}}$ is the total number of beads. ${ }^{53} \mathrm{We}$ can sum the force balance equations (eq 2) for the short arm and long arm of the hooked bead-spring chain. Assuming the chain moves "ropelike", i.e. all sections move at the same velocity $\left(\mathrm{d} x_{1} / \mathrm{d} t\right.$ for the short arm and $\mathrm{d} x_{2} / \mathrm{d} t$ for the long arm), we compute (1) for the short arm

$$
\begin{aligned}
\sum_{n=1}^{j}\left[\zeta_{n}\left(\frac{\mathrm{d} \mathbf{x}_{n}}{\mathrm{~d} t}-\mu \mathbf{E}\right)\right. & \left.=\mathbf{T}_{n}^{\prime}+\mathbf{T}_{n}\right] \\
j \zeta_{n}\left(\frac{\mathrm{d} x_{1}}{\mathrm{~d} t}-\mu E\right) & =-T_{j}^{\prime}
\end{aligned}
$$

and (2) for the long arm

$$
\begin{gathered}
\sum_{n=j+1}^{N_{\mathrm{b}}}\left[\zeta_{n}\left(\frac{\mathrm{d} \mathbf{x}_{n}}{\mathrm{~d} t}-\mu \mathbf{E}\right)=\mathbf{T}_{n}^{\prime}+\mathbf{T}_{n}\right] \\
\left(N_{\mathrm{b}}-j\right) \zeta_{n}\left(\frac{\mathrm{d} x_{2}}{\mathrm{~d} t}-\mu E\right)=-T_{j}^{\prime}
\end{gathered}
$$

We have moved to a $1 \mathrm{D}$ description where $\mathbf{T}_{j}^{\prime}=\mathbf{T}_{j+1}=-T_{j}^{\prime} \mathbf{e}_{x}$ and $\mathbf{e}_{x}=\mu \mathbf{E} /|\mu \mathbf{E}|$. Recall that we have assumed a fully extended unhooking chain. However, if the chain is not fully extended, it will have a nonuniform tension distribution. Note that the details of the tension distribution in each arm are not important at this point because the only surviving tension terms in these above two equations are from the pivot point spring $j$. These equations (eqs 3 and 4) can be subtracted, and using $\mathrm{d} x_{2} / \mathrm{d} t=$ $-\mathrm{d} x_{1} / \mathrm{d} t$ (constant velocity) and $j / N_{\mathrm{b}}=x_{1} / L$, they yield the governing equation for unhooking:

$$
\frac{\mathrm{d} x_{1}}{\mathrm{~d} t}=-\mu E\left(1-\frac{2 x_{1}}{L}\right)
$$

The model unhooking time is found simply by integrating from

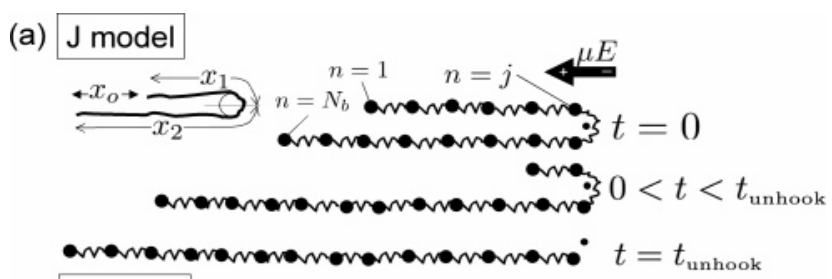

(b) $\mathrm{X}$ model

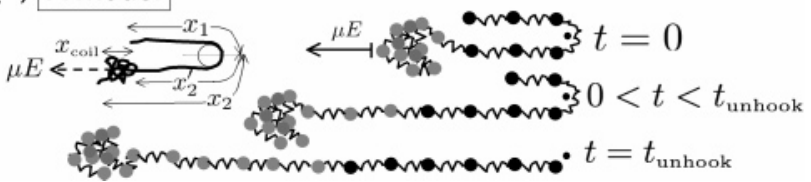

Figure 5. Model schematics at the onset of unhooking. (a) Schematic for the constant-extension $\mathrm{J}$ model. (b) Schematic for the variable extension X model with an extending long arm. The springs have been drawn equal in length to remind the reader of our constant total extension assumption; however, as we show in the text, we do not need to assume this uniform tension distribution.

$t=0$ to $t_{\text {unhook }}$ and $x_{1}=x_{1}(0)$ to 0 :

$$
t_{\text {unhook }}\left(x_{1}(0)\right)=-\frac{L}{2 \mu E} \ln \left(1-\frac{2 x_{1}(0)}{L}\right)
$$

Assuming a uniform distribution of $x_{1}(0)$, this model gives ${ }^{54}$ $\left\langle t_{\text {unhook }}\right\rangle=L /(2 \mu E)$, which scales as $N / E$. At full extension, we also know that $x_{2}-x_{1}=x_{0}$ and $\mathrm{d} x_{\mathrm{o}} / \mathrm{d} t=-2 \mathrm{~d} x_{1} / \mathrm{d} t \cdot{ }^{14}$ By making this substitution in eq 5 , one arrives with the governing equation used in refs 7, 14, and 15 with a linear driving force: $\mathrm{d} x_{0} / \mathrm{d} t=$ $2 \mu E x_{0} / L=2 \lambda E x_{0} / \zeta$ where $\lambda$ is the phenomenological charge per length and $\zeta$ is the molecule's drag coefficient. We have shown here that $\lambda=\mu \xi / L$ for a fully extended chain. We mentioned that previous work applied this model to Rouse simulations and ideal unhooking observations of U/J collisions at high fields, generally with good agreement. However, it needs to be adjusted for our experiments because the DNA do not completely stretch, and some collisions (X collisions) do not have a constant extension. We will consider the modification of finite-Pe moderate stretching first.

At moderate Pe, a tethered chain will not fully extend, and consequently, we do not expect a fully extended hooked configuration. At moderate fields, tethered polymers stretch into configurations resembling "trumpets" and "stem and flowers". 42 We stress that the scaling arguments in ref 42 are for a tethered chain at steady state. However, we know by the continuity of tension at the obstacle pivot point that at least the long arm will not have a steady-state tension distribution. Nevertheless, the configurations at moderate Pe will qualitatively resemble stem and flowers, and we will hypothesize below that a stemand-flower model may be a good way to envision the initial tension distribution in the short arm. To study the unhooking dynamics at constant $\mathrm{Pe}$, we will first still consider constant total extension of the unhooking chain. This model does not require the exact tension distribution in each arm, only that there is an average spring length $l_{\mathrm{s}}$ valid for both arms. The unhooking analysis $^{15}$ is essentially the same as the fully stretched model derived above with the alternate average spring length $l_{\mathrm{S}}$ (so that $\left.N_{\mathrm{b}} l_{\mathrm{s}}=\mathscr{L}\right)$ and altered boundary limits $\left(0<x_{1}<\mathscr{L} / 2\right)$. A sum of bead forces on each arm will give equations equivalent to eqs 3 and 4 , yielding the governing equation

$$
\frac{\mathrm{d} x_{1}}{\mathrm{~d} t}=-\mu E\left(1-\frac{2 x_{1}}{\mathscr{L}}\right)
$$

To arrive at eq 7 , we used the relation $j / N_{\mathrm{b}}=x_{1} / \mathscr{L}$. The 
Table 2. Summary of Hooking Collision Parameters and Results

\begin{tabular}{lccccc}
\hline DNA, Pe & $E(\mathrm{~V} / \mathrm{cm})$ & $\mu E(\mu \mathrm{m} / \mathrm{s})$ & $\mathcal{L}(\mu \mathrm{m})$ & $\tau_{\mathrm{c}}=\mathscr{L} /(\mu E)(\mathrm{s})$ & $\left\langle t_{\mathrm{H}}\right\rangle(\mathrm{s})$ \\
\hline$\lambda, \mathrm{Pe}=2$ & 5.2 & 8.8 & 6.6 & 0.75 & 1.2 \\
$\lambda, \mathrm{Pe}=8$ & 23.4 & 40 & 13 & 0.33 & 0.22 \\
$\mathrm{~T} 4, \mathrm{Pe}=8$ & 8.7 & 14.7 & 45 & 3.1 & 1.8
\end{tabular}

finite-Pe $\mathrm{J}$ model unhooking time is then

$$
\begin{gathered}
t_{\text {unhook }}\left(x_{1}(0)\right)=-\frac{\mathscr{L}}{2 \mu E} \ln \left(1-\frac{2 x_{1}(0)}{\mathscr{L}}\right) \\
=-\frac{\tau_{\mathrm{c}}}{2} \ln \left(1-\frac{2 x_{1}(0)}{\mathscr{L}}\right)
\end{gathered}
$$

where $\tau_{\mathrm{c}}=\mathscr{P} /(\mu E)$. Also note that $\left\langle t_{\text {unhook }}\right\rangle=\tau_{\mathrm{c}} / 2$ for a uniform $x_{1}(0)$ distribution, so that the average unhooking time scales as $N^{\gamma / E}$ where $\gamma$ is the empirical power-law exponent for stretching tethered chains at finite $\operatorname{Pe}(0.54<\gamma<0.75){ }^{30}$

Like a chain at full extension, this model also predicts that the lengths of long and short arms respectively grow and decay exponentially with time. The general solutions are $x_{\mathrm{i}}(t)=\mathcal{L} / 2$ $+\left(x_{i}(0)-\mathscr{L} / 2\right) \exp \left(2 t / \tau_{\mathrm{c}}\right)$ for $i=1,2$. These $\mathrm{J}$ model predictions are plotted by solid gray lines for $\mathrm{U}$ and $\mathrm{J}$ collisions in Figure $4 \mathrm{a}-\mathrm{f}$ with initial conditions $x_{1}(0)=x_{1, \max }$ and $x_{2}(0)$ $=\mathscr{L}-x_{1, \max }$, where $x_{1, \max }$ is the maximum extension of the short arm. Note that for the highly symmetric U collisions the model does not nicely fit actual experimental data; however, it nicely describes the unhooking dynamics of the arms for $\mathrm{J}$ collisions. However, we also apply secondary fits to the $\mathrm{U}$ collision data using arbitrary alternate initial conditions of $x_{1}(0) /$ $\mathscr{L}<0.45$ (dashed gray lines) and find excellent agreement with experimental observations.

The model does a poor job predicting the dynamics of $U$ arms because as $x_{1} / \mathscr{f} \rightarrow 0.5$ arm length fluctuations become important, thereby making the dynamics stochastic instead of deterministic. Consequently, it is clear that there is a transition in $x_{1} / \mathscr{L}$ space from collisions that behave deterministically and collisions greatly affected by fluctuations. An unhooking model with fluctuations has already been proposed in ref 14 and later expanded in ref 41 using a diffusive noise model and a ropeon-pulley first passage time approximation ${ }^{43}$ for the unhooking time. Analysis shows that fluctuations are important in a region of size $\sqrt{D \tau_{\mathrm{c}}} / \mathscr{L}$ in $x_{1}(0) / \mathscr{L}$ space, where $D$ is the DNA's diffusion coefficient. In certain cases, this model nicely describes the observed spread in experimental hold-up times as $x_{1} / \mathscr{L} \rightarrow$ 0.5 ; however, $\mathrm{U}$ collisions are also fairly rare, particularly at $\mathrm{Pe}=8\left(\sqrt{D \tau_{\mathrm{c}}} / \mathscr{L} \sim 0.01\right)$. When looking at initially asymmetric configurations (e.g., with $x_{1}(0) / \mathscr{L}<0.45$ ), the J model nicely predicts the motion of the DNA arms. In the few cases where the model is not perfect, the primary source of error is the variation of model parameters (model predictions are made with parameters $\mathscr{L}$ and $\tau_{\mathrm{c}}$ obtained from the whole ensemble).

$\mathbf{X}$ Model. To model the unhooking time of an X collision, we adopt a new model that is only valid for collisions with small $x_{1}(0)$ and a large arm which continues to grow in length during a collision (Figure $5 \mathrm{~b}$ ). In these $\mathrm{X}$ collisions, we divide the chain into two idealized sections: (1) a freely electrophoresing coil at the end of the long arm and (2) a chain unhooking under tension. We assume the coil to begin a distance $x_{1}(0)$ from the start of the long arm at $t=0$, and $x_{\text {coil }}$ is the size of the coil. This coil lays down contour length as it moves, thereby adding to the time-dependent length of the chain under tension; we denote the long arm length under tension as $x_{2}^{\prime}(t)$ and $x_{2}(t)$ $=x_{2}^{\prime}(t)+x_{\text {coil }}(t)$. We have made this clear in Figure $5 \mathrm{~b}$ by labeling beads that are initially in the coil with gray. The two sections of the long arm move at different velocities; the coil moves at a velocity $\mu E$ whereas the tension bearing section moves at a slower velocity $\left|\mathrm{d} x_{1} / \mathrm{d} t\right|$ (using the ropelike assumption for unhooking). In this picture, the long arm increases in length as fast as the field can move the coil so that $x_{2}(t)=$ $x_{1}(0)+x_{\text {coil }}(0)+\mu E t$.

Returning to eqs 3 and 4, we can sum the contribution from tension-bearing springs (all springs not in the leading coil). For the short arm

$$
j \zeta_{n}\left(\frac{\mathrm{d} x_{1}}{\mathrm{~d} t}-\mu E\right)=-T_{j}^{\prime}
$$

and for the long arm

$$
\left(j(0)+\frac{\mu E t}{l_{\mathrm{s}}}\right) \zeta_{n}\left(\frac{\mathrm{d} x_{1}}{\mathrm{~d} t}-\mu E\right)=-T_{j}^{\prime}
$$

Recall $l_{\mathrm{s}}$ is the average spring length of the bead-spring model so $j(0)+\mu E t / l_{\mathrm{s}}$ is the number of beads in the long arm under tension if we assume $\mathrm{d} x_{\text {coil }} / \mathrm{d} t \ll \mathrm{d} x_{2} / \mathrm{d} t$. Using the relations $j(0) l_{\mathrm{s}}$ $=x_{1}(0)$ and $j l_{\mathrm{s}}=x_{1}$ and subtracting these two equations yields

$$
\left(x_{1}(0)+\mu E t+x_{1}\right) \frac{\mathrm{d} x_{1}}{\mathrm{~d} t}=-\mu E\left(x_{1}(0)+\mu E t-x_{1}\right)
$$

The governing equation of the $\mathrm{X}$ model (eq 11) is a nonlinear, nonautonomous differential equation. This equation can be nondimensionalized, using $\tilde{x}_{1}=x_{1} / x_{1}(0)$ and $\tilde{t}=\mu E t / x_{1}(0)$ :

$$
\frac{\mathrm{d} \tilde{x}_{1}}{\mathrm{~d} \tilde{t}}=\frac{-1-\tilde{t}+\tilde{x}_{1}}{1+\tilde{t}+\tilde{x}_{1}}
$$

In this nondimensional equation, all initial conditions map to $\tilde{x}_{1}(0)=1 . \tilde{x}_{1}(\tilde{t})$ falls as $\tilde{t}$ increases, from a slope of 0 to -1 until $\tilde{x}_{1}\left(\tilde{t}_{\text {unhook }}\right)=0$. Note that now the condition $\tilde{x}_{1}\left(\tilde{t}_{\text {unhook }}\right)=0$ is independent of the initial condition $x_{1}(0)$. Using Euler's method, we numerically solve for when $\tilde{x}_{1}\left(\tilde{t}_{\text {unhook }}\right)=0$ and find that $\tilde{t}_{\text {unhook }} \simeq 2$.1. Converting back to dimensional variables, we see that the $\mathrm{X}$ model trapping time is a linear function of the initial short arm length $x_{1}(0)$ :

$$
t_{\text {unhook }}\left(x_{1}(0)\right) \simeq 2.1 \frac{x_{1}(0)}{\mu E}
$$

For this $\mathrm{X}$ model to be physically consistent, $x_{1}(0) / \mathscr{L}<0.3$. If this condition fails, the coil completely unwinds during unhooking. We will see that very few $\mathrm{X}$ collisions have $x_{1}(0) / \mathscr{L}>$ 0.3 ; however, we must stress that this model is most useful for small short arms.

We can apply the numerical solution $x_{1}(t)$ for eq 11 to the observed T4 DNA X collisions at Pe $=8$ in Figure $4 \mathrm{~g}-\mathrm{i}$. We plot this numerical solution along with the $\mathrm{X}$ model prediction of $x_{2}(t)=x_{2}(0)+\mu E t$ in solid gray in Figure $4 \mathrm{~g}-\mathrm{i}$ beginning at the maximum value of $x_{1}$ and using the same experimentally measured values of $\mathscr{f}$ and $\mu E$. The $\mathrm{X}$ model very nicely predicts the observed time dependence of both arms, though as $x_{1}(0) / \mathscr{L} \rightarrow 0.3, x_{2}$ may slightly drop below the idealized coil unwinding prediction of the model near the end of the unhooking time (Figure 4i).

The Short Arm. Here we begin our attempt to determine how these models can help predict the experimental center-ofmass hold-up times $\left(t_{\mathrm{H}}\right)$. The hold-up time is more important to study for hooking-based applications like DNA mapping which require separation (i.e., center-of-mass displacement) of 


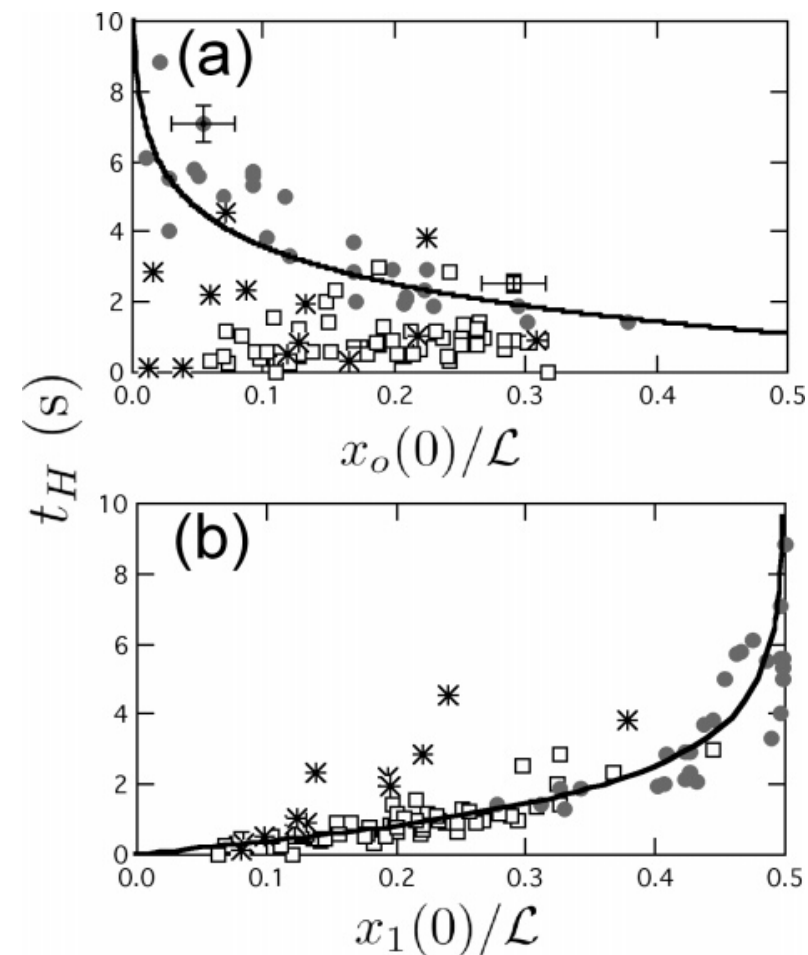

Figure 6. (a) Plot of the collision time as a function of the difference in arm lengths $x_{0}$ (scaled by the constant $\mathrm{U} / \mathrm{J}$ extension value $\mathscr{L}$ ) at the onset of unhooking for T4 DNA at $\mathrm{Pe}=8$. U/J collisions are shown as solid circles, $\mathrm{X}$ collisions are shown as open squares, and $\mathrm{W}$ collisions are shown as stars. The line is the $\mathbf{J}$ model for a chain at constant extension (eq 14). Characteristic error bars for long and short collision times are also shown. (b) Plot of the collision time as a function of the short arm length at the onset of unhooking (scaled by the constant $\mathrm{U} / \mathrm{J}$ extension value $\mathscr{f}$ ). The line is the rope-on-pulley $t_{\text {unhook }} \mathrm{J}$ model based on the short arm extension (eq 8).

individual molecules. We know that the unhooking time is the primary component to the total hold-up time, so we will first directly compare our unhooking time model predictions to experimental hold-up time results. However, we should stress beforehand that we know $t_{\mathrm{H}} \neq t_{\text {unhook }}$ because of unraveling and other effects to be discussed below. Nevertheless, this comparison between experimental data and model predictions will provide some important physical insight and raise some interesting questions.

We compare the $\mathbf{J}$ model to our experimental data in two ways: first plotting the measured hold-up time $t_{\mathrm{H}}$ vs $x_{0}(0)$ and second plotting $t_{\mathrm{H}}$ vs $x_{1}(0)$. We expect eq 8 to nicely model the $\mathrm{U} / \mathrm{J}$ collision unhooking time (and hence hold-up time) as a function of $x_{1}(0)$. Similarly, using the relation $\mathscr{Z}-2 x_{1}(0)=$ $x_{0}(0)$ for a constant-extension unhooking chain, eq 8 can be rewritten as

$$
t_{\text {unhook }}\left(x_{0}(0)\right)=-\frac{\tau_{\mathrm{c}}}{2} \ln \left(\frac{x_{0}(0)}{\mathscr{L}}\right)
$$

which would predict unhooking times for U/J collisions as a function of $x_{0}(0)$. For comparison of the model to our data, we have split our ensemble into U/J collisions (solid circles), $\mathrm{X}$ collisions (open boxes), and $\mathrm{W}$ collisions (stars). To obtain $x_{0}(0)$ and $x_{1}(0)$, we manually perform length measurements at the instant the short arm begins to retract. Figure 6a shows the experimental hold-up times vs $x_{0}(0)$, and Figure $6 \mathrm{~b}$ shows the same hold-up time data plotted against $x_{1}(0)$. Figure 6a clearly shows that the $\mathrm{J}$ model for a moderately extended DNA (eq 14) does nicely predict the hold-up times of many observed constant- $\mathscr{\complement} \mathrm{U}$ and $\mathrm{J}$ collisions. The observed hold-up times are dispersed around the model prediction, likely due to thermal fluctuations of the arms. However, as expected, it is obvious that the model cannot predict the hold-up time of the many $\mathrm{X}$ (and possibly W) collisions. Since W events are rare, we neglect them in the bulk of the remaining discussion. It is striking though that Figure $6 \mathrm{~b}$ shows that the constant extension $\mathrm{J}$ model adequately describes all the $X$ and U/J collisions when plotted against $x_{1}(0)$. The close agreement between the predicted unhooking time and all observed hold-up times suggests that the short arm mediates the unhooking process. We can explain this result by examining the anticipated tension distribution of the hooked DNA chain.

As mentioned above, we assume a U or J DNA molecule to have a constant extension while unhooking. This implies the tension distribution is more or less frozen during the unhooking process. More likely there is a complex competition between tension relaxation and the electrical driving force. However, what is this tension distribution at the onset of unhooking? During the unraveling, the short arm may eventually reach steady extension even if the long arm is still extending. Observation of single DNA configurations (both J's and X's) during unhooking supports this hypothesis. We can model the short arm in both $\mathrm{U} / \mathrm{J}$ and $\mathrm{X}$ collisions at the onset of unhooking as an idealized tethered chain with a steady-state tension profile in a uniform field. However, the long arm will have an unsteady tension profile. The slight imbalance in the growing tension of the long arm will then begin to pull the short arm around the obstacle. Consequently, the magnitude of the "tethering force" $T_{j}^{\prime}$ during unhooking is set by the size of the short arm, which we hypothesize reaches a steady-state tension distribution at the onset of unhooking. The main consequence is that a $\mathrm{J}$ collision and an X collision with the same short arm lengths can be modeled to have approximately the same hold-up times.

\section{Hold-Up Time: Center-of-Mass and Unraveling Effects}

We have just derived microscopic models for $t_{\text {unhook }}$ and have shown that they nicely predict observed arm dynamics during the unhooking process. Furthermore, we have noticed that when formulating the $\mathrm{J}$ model in terms of the short arm length, it adequately predicts hold-up times $t_{\mathrm{H}}$ for even $\mathrm{X}$ collisions. However, we now look at this result from a single molecule standpoint to determine why we observe this nearly universal agreement with the $\mathrm{J}$ model. Specifically, we must address how these unhooking models can predict the experimentally observable center-of-mass hold-up time $t_{\mathrm{H}}$.

Figure 7 shows a schematic of a typical center of mass trajectory during a hooking collision. Recall that $t_{\text {unrav }}$ is the time between initial impact and the onset of unhooking and $t_{\text {unhook }}$ is the time to unhook off of the obstacle after unraveling. Also recall that $t_{\mathrm{H}}$ is the time offset between linear fits to the approach and release data ${ }^{19}$ and has contributions from unraveling and unhooking. However, it is clear from Figure 7 that $t_{\mathrm{H}}$ $\neq t_{\text {unhook }}+t_{\text {unrav }}$ because the center of mass moves during these events. As the DNA unravels, the center of mass moves from the obstacle position at $x=0$ to a position downfield at the onset of unhooking $(t=0)$. But as the DNA unhooks, the center of mass continues to move downfield. We define the distance the center of mass moves during unhooking as $\Delta x_{\text {com. }}$ The overall effect of the center-of-mass motion is that the hold-up time becomes equal to

$$
t_{\mathrm{H}}=t_{\text {unhook }}+\delta t_{1}-\delta t_{2}
$$

where $\delta t_{1}$ accounts for unraveling and $\delta t_{2}$ accounts for center- 


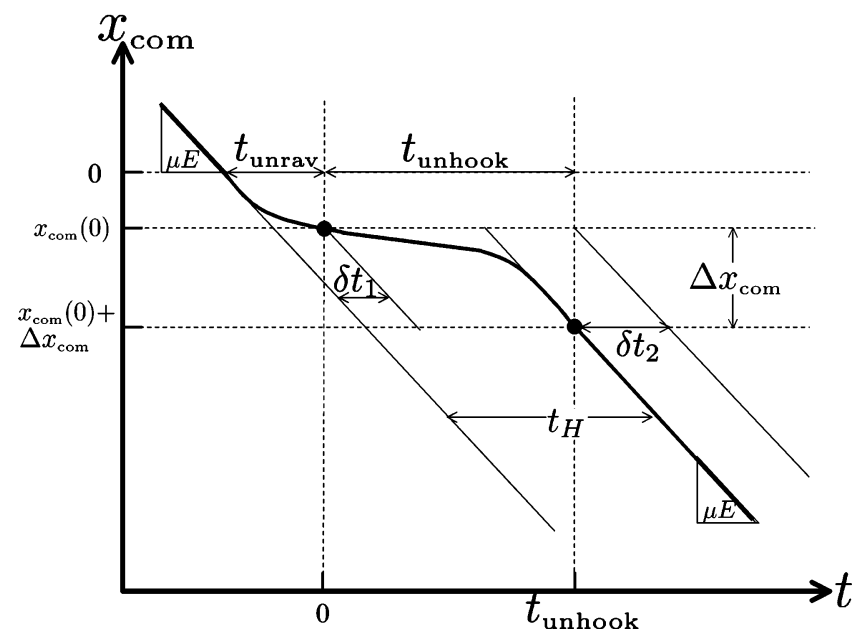

Figure 7. A schematic of the typical center-of-mass trace for a hooking collision.

of-mass motion during unhooking. We will now present idealized models to predict $\delta t_{1}$ and $\delta t_{2}$ for the $\mathrm{J}$ and $\mathrm{X}$ models. Below, we will then modify the $\mathrm{J}$ and $\mathrm{X}$ models to account for $\delta t_{1}$ and $\delta t_{2}$, referring to these new models for $t_{\mathrm{H}}$ as "modified".

Modified J Model. To model center-of-mass movement and unraveling effects for $\mathrm{U} / \mathrm{J}$ collisions, we will consider a constant extension DNA with uniform mass density along its length, i.e., the ideal rope approximation. This is an oversimplication as the ends of the DNA do generally appear brighter than the stretched portions. However, this analysis is a satisfying first approximation and even provides a worst-case limit for the magnitude of each of these effects because brighter ends would decrease the perturbations $\delta t_{1}$ and $\delta t_{2}$.

First we determine the hold-up time due to unraveling $\delta t_{1}$ for the ideal rope approximation. We know from the $\mathrm{J}$ model that $x_{\text {com }}(0)=-\mathscr{L}\left(1 / 4+\left(x_{1}(0) / \mathscr{L}-1 / 2\right)^{2}\right)$. We assume that the ideal rope takes time $t_{\text {unrav }}=\left(\mathscr{L}-x_{1}(0)\right) /(\mu E)$ to reach this initial condition for unhooking from the onset of collision with the obstacle at $x_{\text {com }}=0$. Consequently, we can use simple graphical methods (Figure 7) to determine the additional holdup time for unraveling:

$$
\delta t_{1}=\tau_{\mathrm{c}}\left(\frac{1}{2}-\frac{x_{1}^{2}(0)}{\mathscr{L}^{2}}\right)
$$

A similar geometric method can be used to determine $\delta t_{2}$. Again, we know the initial center-of-mass position $x_{\text {com }}(0)$ of the ideal unhooking rope; however, from the uniform mass density assumption, we also know that $x_{\text {com }}\left(t_{\text {unhook }}\right)=-\mathscr{P} / 2$ for an ideal rope. Consequently, $\Delta x_{\text {com }}=-\mathscr{L} / 2+x_{\text {com }}(0)$, and the hold-up time lost due to center-of-mass motion for an ideal rope $\delta t_{2}$ is

$$
\delta t_{2}=\tau_{\mathrm{c}}\left(\frac{x_{1}(0)}{\mathscr{L}}-\frac{x_{1}^{2}(0)}{\mathscr{L}^{2}}\right)
$$

Finally, we can determine the hold-up time $t_{\mathrm{H}}$ for the modified $\mathrm{J}$ model by adding eqs 8 and 16 and subtracting eq 17 :

$$
t_{\mathrm{H}}=\tau_{\mathrm{c}}\left[\frac{-1}{2} \ln \left(1-\frac{2 x_{1}(0)}{\mathscr{L}}\right)+\frac{1}{2}-\frac{x_{1}(0)}{\mathscr{L}}\right]
$$

Note that the experimentally observed $t_{\mathrm{H}}$ will be slightly longer than $t_{\text {unhook }}$; however, the two times will converge to the same value as $x_{1}(0) / \mathscr{L} \rightarrow 1 / 2$.
Modified X Model. The anticipated perturbations to the observed hold-up time can also be computed geometrically for the $\mathrm{X}$ model. The increase from unraveling is now limited to the time it takes to extend the short arm, $t_{\text {unrav }}=x_{1}(0) / \mu E$, but must likewise include the effects of center-of-mass motion during unraveling. We approximate the center of mass of the coil section of an $\mathrm{X}$ collision as residing at the end of the long arm's rope section $\left(x_{2}^{\prime}\right)$. Consequently, the center of mass at the onset of unhooking is

$$
x_{\mathrm{com}}(0) \simeq\left[\frac{x_{1}(0)^{2}}{\mathscr{L}}+\left(1-\frac{2 x_{1}(0)}{\mathscr{L}}\right) x_{1}(0)\right]
$$

Using geometric relations as in the modified $\mathrm{J}$ model, the additional unraveling time for the modified $\mathrm{X}$ model is

$$
\delta t_{1}=\tau_{\mathrm{c}} \frac{x_{1}(0)^{2}}{\mathscr{\rho}^{2}}
$$

The lost hold-up time from center-of-mass motion during unhooking for an $\mathrm{X}$ collision is $\Delta x_{\text {com }} / \mu E$, where $\Delta x_{\text {com }}$ is the distance the center of mass moves downfield during unhooking. Using $x_{2}^{\prime}(t) \simeq \mu E t+x_{1}(0)$ and eq 13 , the center of mass at the end of unhooking for an $\mathrm{X}$ collision is

$$
x_{\text {com }}\left(t_{\text {unhook }}\right) \simeq\left[4.8 \frac{x_{1}(0)^{2}}{\mathscr{L}}+3.1 x_{1}(0)\left(1-3.1 \frac{x_{1}(0)}{\mathscr{L}}\right)\right]
$$

Subtracting eq 19 from 21 gives $\Delta x_{\text {com }} \simeq 2.1 x_{1}(0)-3.8 x_{1}^{2}(0) /$ $\mathscr{L}$. Consequently, the $\mathrm{X}$ model center-of-mass hold-up time due to center-of-mass motion during unhooking is

$$
\delta t_{2}=\tau_{\mathrm{c}}\left(2.1 \frac{x_{1}(0)}{\mathscr{L}}-3.8 \frac{x_{1}(0)^{2}}{\mathscr{L}^{2}}\right)
$$

Finally, we can determine the hold-up time $t_{\mathrm{H}}$ for the modified $\mathrm{X}$ model by adding eqs 13 and 20 and subtracting eq 22:

$$
t_{\mathrm{H}}=4.8 \tau_{\mathrm{c}} \frac{x_{1}(0)^{2}}{\mathscr{P}^{2}}
$$

By comparing eq 23 to the unhooking time model eq 13, we see that adding unraveling and center-of-mass effects changes the functionality of the collision time with the short arm length (from linear to quadratic).

Comparison to Experiments. Because we have access to the experimental center-of-mass trajectories of all impacting DNA in an ensemble, we can compare some of the modified hold-up time model predictions with actual experimental data. Figure 8a,b shows the center-of-mass trajectories during a typical $\mathrm{J}$ and $\mathrm{X}$ collision. The markers switch from closed to open when a molecule completely unhooks. Obtaining precise center-ofmass measurements requires a strong signal-to-noise ratio for the entire DNA's fluorescent cloud. However, one limitation of these experiments is that the signal-to-noise ratio drops when the DNA is stretched. This can be seen in Figure 8c,d, which shows the total integrated fluorescence cloud intensity of the each of the DNA molecules in Figure 8a,b. As expected, the intensity plateaus gradually drop from preimpact to postunhooking due to photobleaching. But notice that the intensity signal drops significantly when the DNA is on the obstacle. The low signal values of stretched sections of the DNA would shift the calculated center of mass to the leading end of the long arm because that is where the signal is generally the 


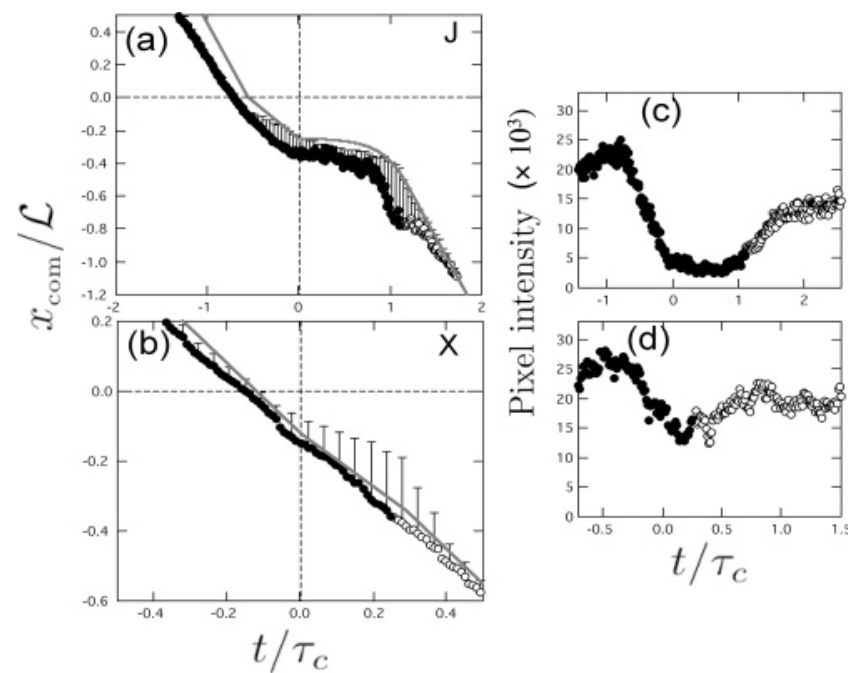

Figure 8. Experimental center-of-mass trajectories of (a) a J collision and (b) an $\mathrm{X}$ collision. The solid gray lines are model predictions (we connect initial and final center-of-mass coordinates during unraveling; during unhooking we use eq 24 for the $\mathrm{J}$ model and a numerical solution to eqs 12 and 25 for the $\mathrm{X}$ model). Unidirectional error bars correspond to the horizontal distance between the actual center of mass and the middle of the DNA fluorescence cloud. (c, d) The integrated intensity of the DNA's fluorescence cloud (after noise filtering) as a function of time scaled by $\tau_{\mathrm{c}}$ for (c) a J collision and (d) an $\mathrm{X}$ collision.

strongest. Consequently, a limitation of these experiments is that the uncertainty in the center-of-mass calculation grows when the molecule is extended. Therefore, we add unidirectional error bars to the center-of-mass plots as the difference between the calculated center of mass and the middle of the DNA's fluorescence cloud. These error bars serve as a maximum estimate of error in the low-signal center-of-mass calculation. Despite the moderate uncertainty in the center-of-mass position of hairpin-shaped configurations, the center of mass of DNA coils moving away from the obstacle is very precise. Recall that it is this more precise data (approach and release trajectories) that we use to compute $t_{\mathrm{H}}$.

Returning to Figure 8a,b, we can compare the observed center-of-mass trajectories with those predicted from the ideal rope $\mathrm{J}$ model and the $\mathrm{X}$ model. First we look at the $\mathrm{J}$ collision in Figure 8a. According to the ideal rope approximation (gray line), the center of mass will move at constant velocity $\mu E$ until the DNA impacts the obstacle. Then it will slow down as the DNA unravels. Knowing the initial and final points for unraveling, we will simply connect these points with a line in Figure $8 \mathrm{a}$. Then the ideal rope will unhook as

$$
x_{\mathrm{com}}(t)=\frac{x_{1}^{2}(t)+x_{2}^{2}(t)}{2 \mathscr{L}}
$$

which will depend the initial symmetry $\left(x_{1}(0)\right)$ of the unhooking configuration. Note that for the shown example this corresponds to a slowly decreasing plateau in $x_{\text {com }}$ followed by a sharply decreasing section at the end of unhooking. After this drop, the center of mass will then move away from the obstacle at constant velocity $\mu E$.

By comparing this anticipated motion for an ideal rope to actual data in Figure 8a, we see that the observed center of mass is generally further downfield (lower on plot) than predicted by the ideal rope approximation. This is due to the "stem-andflower-like" 42 configuration of the unhooking arms. However, the ideal rope model does capture the important aspects of the center-of-mass motion. We observe both the slight slowdown of $x_{\text {com }}$ during unraveling and the characteristic plateau during unhooking. However, we also observe an anomalous secondary plateau that begins after the DNA has left the obstacle. This observation is general to the majority of $\mathrm{U} / \mathrm{J}$ collisions; however, there is no physical reason for the DNA's center of mass to slow down during relaxation. This secondary plateau is an artifact of the low signal and stems from the increasing fluorescence signal of the relaxing DNA molecule. The signal increases as the DNA relaxes because the monomer density per pixel is increasing. When accounting for the error in the centerof-mass calculation of a hooked configuration, the observed center-of-mass dynamics nearly parallel the ideal rope model except for a constant downfield displacement. Consequently, there is hope that a simplified model like the modified $\mathbf{J}$ model can accurately predict center-of-mass hold-up times.

In the same way, we look at a typical X collision center-ofmass trajectory in Figure 8b. An idealized X collision would approach the obstacle at constant velocity, slightly slow down during unraveling and unhooking, and then move away from the obstacle at constant velocity. As for the modified J model, we can consider the average velocity of the center of mass during unraveling and how the center of mass will move during unhooking by using the numerical solution to the modified $\mathrm{X}$ model (eq 12) and arrive at

$$
x_{\mathrm{com}}(t) \simeq \frac{x_{1}{ }^{2}(t)+x_{2}^{\prime 2}(t)}{2 \mathscr{P}}+\left(1-\frac{x_{1}(t)}{\mathscr{L}}-\frac{x_{2}^{\prime}(t)}{\mathscr{L}}\right) x_{2}^{\prime}(t)
$$

In comparison with the $\mathbf{J}$ model, we see closer agreement between the experimental data and model predictions. The closer agreement stems from the better signal-to-noise ratio of $\mathrm{X}$ collisions and from the more accurate depiction of the mass density distribution in the modified $\mathrm{X}$ model. But like the modified $\mathrm{J}$ model, the modified $\mathrm{X}$ model also nicely predicts the timing of the different stages of the center-of-mass trajectory.

To continue our single molecule analysis and experimental verification of the hold-up time model, we extract $t_{\text {unhook }}, \delta t_{1}$, and $\delta t_{2}$ directly from each collision in the experimental ensemble. To determine $t_{\text {unhook }}$, we manually determine the total time each hooking DNA molecule remains on the obstacle from the onset of unhooking (when $x_{1}$ reaches a maximum) until the DNA leaves the obstacle. Figure 9a compares experimentally determined center-of-mass hold-up times for $\mathrm{T} 4$ at $\mathrm{Pe}=8$ against the manually determined experimental unhooking time. $\mathrm{W}$ collisons are not included, and again $\mathrm{X}$ collisions are represented as open boxes and $\mathrm{U} / \mathrm{J}$ collisions are filled circles. The black line is the equation $t_{\mathrm{H}}=t_{\text {unhook }}$, and the gray line is the modified X model (substituting eq 13 into eq 23). Note the apparent switch in power-law functionality between $\mathrm{X}\left(t_{\mathrm{H}} \sim\right.$ $\left.t_{\text {unhook }}^{2}\right)$ and $\mathrm{J}\left(t_{\mathrm{H}} \sim t_{\text {unhook }}\right)$ hold-up times which supports our models. Furthermore, using both the manually determined beginning and ending frames of a collision and the line fits for the approach and release center-of-mass data, we then used automated code to extract $\delta t_{1}$ and $\delta t_{2}$. These calculations do not rely on any of the "low signal" center-of-mass data often seen in some highly extended U/J configurations. Figure $9 b$ compares the experimental hold-up times to the experimentally derived $t_{\text {unhook }}+\delta t_{1}-\delta t_{2}$. The black line is the equation $t_{\mathrm{H}}=$ $t_{\text {unhook }}+\delta t_{1}-\delta t_{2}$. The satisfactory agreement of this line with the data verifies both our data analysis techniques and the importance of center-of-mass motion and unraveling perturbations, particularly for $\mathrm{X}$ collisions. 

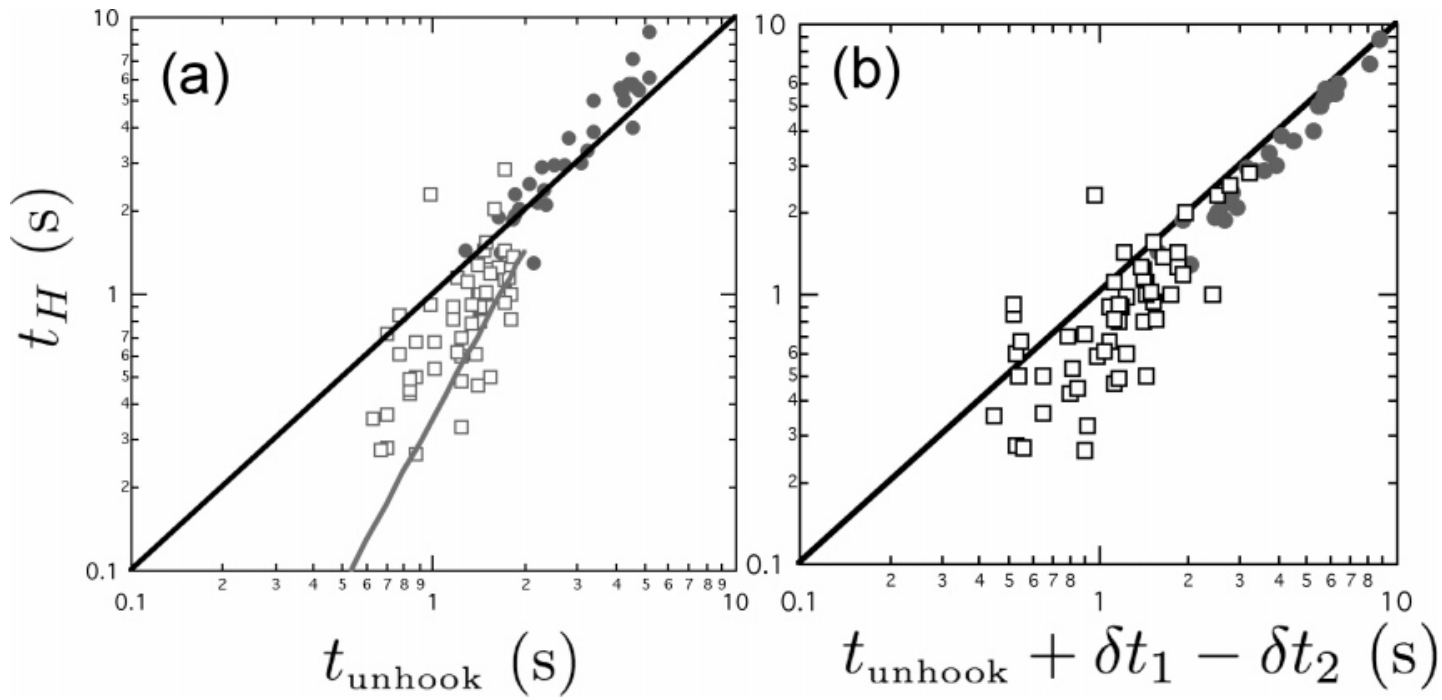

Figure 9. (a) Experimental comparison of $t_{\mathrm{H}}$ and $t_{\text {unhook }}$ for T4 DNA at Pe $=8$. The solid black line is $t_{\mathrm{H}}=t_{\text {unhook }}$, and the solid gray line is the modified X model (eqs 23 and 13). Filled circles correspond to U/J collisions whereas open boxes correspond to X collisions. (b) Experimental comparison of $t_{\mathrm{H}}$ and $t_{\text {unhook }}+\delta t_{1}-\delta t_{2}$. The solid black line is $t_{\mathrm{H}}=t_{\text {unhook }}+\delta t_{1}-\delta t_{2}$.

\section{Hold-Up Times for Variable DNA Size and Field Strength}

The preceding single molecule collision case study for T4 DNA at $\mathrm{Pe}=8$ showed how the unhooking dynamics relate to center-of-mass hold-up times. T4 was chosen because it is long enough to easily resolve and track the arm motion. Here we will use the models and insight developed in the example above to study the center-of-mass hold-up times for smaller DNA at alternate fields ( $\lambda$ DNA at $\mathrm{Pe}=2$ and 8 ).

Figure $10 \mathrm{a}-\mathrm{c}$ shows experimental $t_{\mathrm{H}}$ data as a function of the initial short arm length for $\lambda$-DNA at $\mathrm{Pe}=2$ and 8 as well as T4 DNA at $\mathrm{Pe}=8$. Recall from Figure 6 that plotting $t_{\mathrm{H}}$ against the short arm length collapses these data, which we attributed to the fact that the short arm governs the tension at the obstacle pivot. In Figure $10 \mathrm{a}-\mathrm{c}$ we include the $\mathrm{J}$ unhooking time model (eq 8 , thick black line), the modified J holdup time model (eq 18, thick dashed curve), the X unhooking time model (eq 13, thin red line), and the modified X hold-up time model (eq 23, thin red dashed curve). These models have different regimes of validity, and we would expect most $\mathrm{X}$ collisions to follow the lower thin red dashed curves whereas most $\mathbf{J}$ collisions would follow the black dashed line. However, the $\mathrm{J}$ unhooking time model adequately fits both types of collision data for all three data sets. As we saw with our in depth analysis of the T4 data, this agreement is mostly a coincidence because the actual center-of-mass hold-up time depends on the unraveling and center-of-mass motion perturbations. However, these perturbations nearly cancel for most U/J collisions and yield a function close to the $\mathrm{J}$ model for $\mathrm{X}$ collisions.

It is interesting to note that no $\mathrm{U} / \mathrm{J}$ collisions were classified with $x_{1}(0) / \mathscr{L}<0.25$, presumably due to the large difference in time it takes for the long arm (as opposed to the short arm) to completely unravel. In the regime $x_{1}(0) / \mathscr{L}>0.25$, the $\mathrm{J}$ model and the modified $\mathbf{J}$ model are nearly equivalent. Furthermore, note the spread of the U/J hold-up times around the model prediction grows as Pe is reduced from 8 to 2 . This is likely due to the increased importance of Brownian fluctuations on unhooking configurations at low Pe and may be explored by incorporating a noise term in the governing equation for unhooking (eq 2). ${ }^{14,41}$
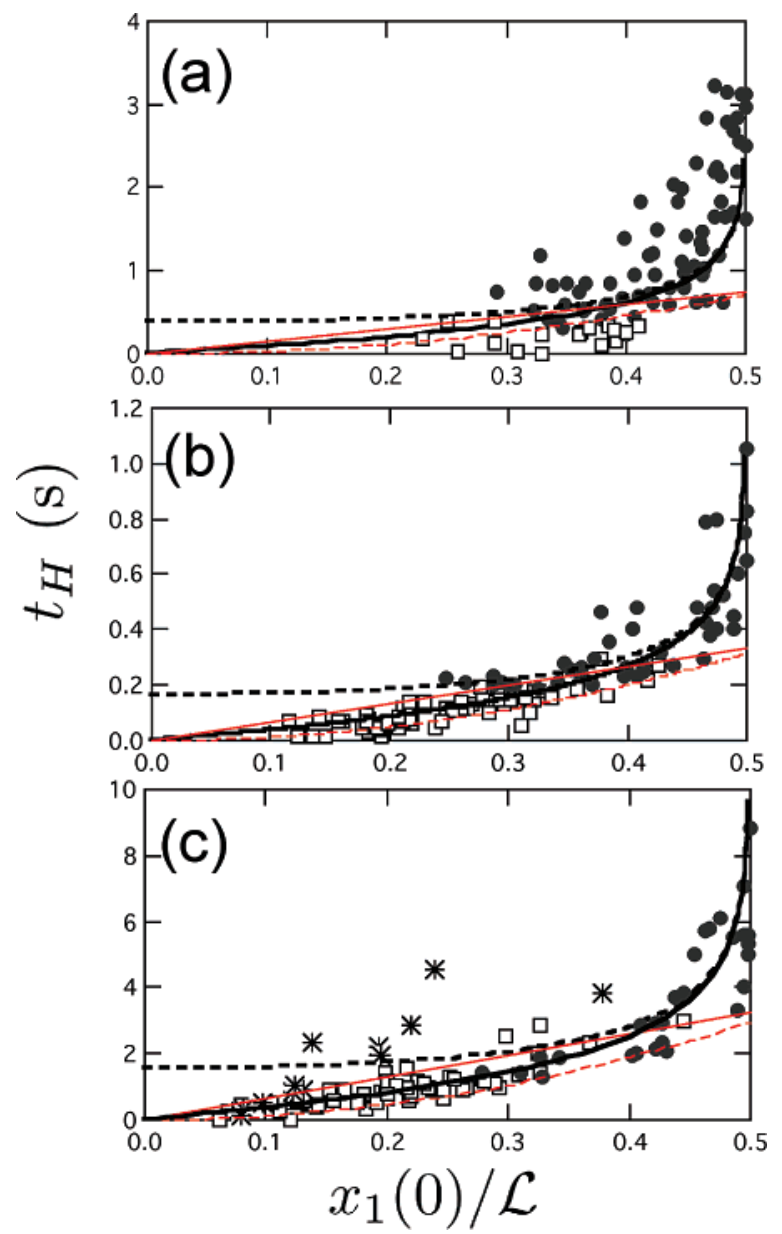

Figure 10. Experimental test of collision time models for multiple DNA sizes and field strengths. Plots of the collision time as a function of the short arm length at the onset of unhooking (scaled by the constant $\mathrm{U} / \mathrm{J}$ extension value $\mathscr{L}$ ) for (a) $\lambda$-DNA at $\mathrm{Pe}=2$, (b) $\lambda$-DNA at $\mathrm{Pe}=$ 8 , and (c) T4 DNA at $\mathrm{Pe}=8$. U/J collisions are shown as solid circles, $\mathrm{X}$ collisions are shown as open squares, and $\mathrm{W}$ collisions are shown as stars. The black line in each plot is the $t_{\text {unhook }} \mathrm{J}$ model based on the short arm extension (eq 8), and the dashed line is the modified $\mathrm{J}$ model (eq 18) that includes corrections from unraveling and center-of-mass motion. The thin red line is the $\mathrm{X}$ model prediction for $t_{\text {unhook }}\left(x_{1}(0)\right)$ (eq 13), and the red dashed line is the modified X model prediction for $t_{\mathrm{H}}\left(x_{1}(0)\right)$ (eq 23). 


\section{Conclusions and Outlook}

In this study, we systematically analyzed the dynamics of single DNA molecules driven by an electric field and hooking on a small microfabricated post. Hooking collisions can lead to size-dependent DNA separations, so we examined the unhooking dynamics which dominate the total hold-up time during a collision. We classified the collisions and developed models to predict the unhooking dynamics and ensuing collision times. The most striking result from our work is that we determined that a new type of collision, which we called an $\mathrm{X}$ (extending) collision, is statistically very probable and behaves very differently than a classic rope-on-a-pulley. From collapse of the data and our models, we introduced the notion that the short arm of a hooked DNA generally governs the release dynamics. Consequently, X collisions and J collisions with the same short arm length have similar unhooking times. This equivalence was explicitly demonstrated in single molecule observations and using simplified models for perturbations to the hold-up time for unraveling and center-of-mass motion. Therefore, a rope-on-pulley model based on the short arm length dynamics of a constant extension chain can nicely predict the observed collision time data for a majority of the impact ensemble.

While here we only considered DNA collision with a single post, the various classes of collisions we found should still be present when examining post arrays ${ }^{12-14,25,44}$ and be considered in future modeling efforts..$^{23-25}$ In a broader context, similar disentanglement processes occur in polymers flowing over a polymer brush,${ }^{45}$ DNA separations using dilute neutral polymers as a sieving medium, ${ }^{46}$ and flows of polymer solutions ${ }^{47}$ Finally, in direct linear analysis, ${ }^{48,49}$ DNA collisions with posts may be a useful way of preconditioning DNA configurations before attempting to completely stretch DNA in an elongational flow ${ }^{48-50}$ or electric field. ${ }^{28,51}$

Acknowledgment. This work was supported by NSF CAREER Grant CTS-0239012.

\section{References and Notes}

(1) Starkweather, M. E.; Muthukumar, M.; Hoagland, D. A. Macromolecules 1998, 31, 5495 .

(2) Starkweather, M.; Muthukumar, M.; Hoagland, D. Macromolecules 1999, 32, 6837.

(3) Doyle, P. S.; Ladoux, B.; Viovy, J.-L. Phys. Rev. Lett. 2000, 84, 4769

(4) Doi, M.; Edwards, S. F. The Theory of Polymer Dynamics; Clarendon Press: Oxford, UK, 1988.

(5) Perkins, T. T. Exploring Polymer Dynamics with Single DNA Molecules. Ph.D. Thesis, Stanford University,1997.

(6) Shaqfeh, E. S. G. J. Non-Newtonian Fluid Mech. 2005, 130, 1.

(7) Song, L.; Maestre, M. F. J. Biomol. Struct. Dyn. 1991, 9, 87.

(8) Deutsch, J. M. Phys. Rev. Lett. 1987, 59, 1255.

(9) Deutsch, J. M. Science 1988, 240, 922.

(10) Deutsch, J. M.; Madden, T. L. J. Chem. Phys. 1989, 90, 2476.

(11) Deutsch, J. M. J. Chem. Phys. 1989, 90, 7436.

(12) Volkmuth, W. D.; Austin, R. H. Nature (London) 1992, 358, 600

(13) Austin, R. H.; Volkmuth, W. D. Analusis 1993, 21, 235.

(14) Volkmuth, W. D.; Duke, T.; Wu, M. C.; Austin, R. H.; Szabo, A. Phys. Rev. Lett. 1994, 72, 2117.

(15) Sevick, E. M.; Williams, D. R. M. Phys. Rev. E 1994, 50, R3357.

(16) Nixon, G. I.; Slater, G. W. Phys. Rev. E 1994, 50, 5033.
(17) Sevick, E. M.; Williams, D. R. M. Phys. Rev. Lett. 1996, 76, 2595.

(18) André, P.; Long, D.; Ajdari, A. Eur. Phys. J. B 1998, 4, 307.

(19) Saville, P. M.; Sevick, E. M. Macromolecules 1999, 32, 892.

(20) Patel, P. D.; Shaqfeh, E. S. G. J. Chem. Phys. 2003, 118, 2941.

(21) Minc, N.; Fütterer, C.; Dorfman, K. D.; Bancaud, A.; Gosse, C.; Goubault, C.; Viovy, J.-L. Anal. Chem. 2004, 76, 3770.

(22) Kaji, N.; Tezuka, Y.; Takamura, Y.; Ueda, M.; Nishimoto, T. Nakanishi, H.; Horiike, Y.; Baba, Y. Anal. Chem. 2004, 76, 15.

(23) Dorfman, K. D.; Viovy, J.-L. Phys. Rev. E 2004, 69, 011901.

(24) Minc, N.; Viovy, J.-L.; Dorfman, K. D. Phys. Rev. Lett. 2005, 94 198105.

(25) Minc, N.; Bokov, P.; Zeldovich, K. B.; Fütterer, C.; Viovy, J.-L.; Dorfman, K. D. Electrophoresis 2005, 26, 362.

(26) Randall, G. C.; Doyle, P. S. Phys. Rev. Lett. 2004, 93, 058102.

(27) Olson, D. J.; Johnson, J. M.; Patel, P. D.; Shaqfeh, E. S. G.; Boxer, S. G.; Fuller, G. G. Langmuir 2001, 17, 7396.

(28) Randall, G. C.; Doyle, P. S. Macromolecules 2005, 38, 2410

(29) Long, D.; Viovy, J.-L.; Ajdari, A. J. Chem. Phys. 2003, 118, 2941

(30) Perkins, T. T.; Smith, D. E.; Larson, R. G.; Chu, S. Science 1995, $268,83$.

(31) Xia, Y.; Whitesides, G. M. Angew. Chem., Int. Ed. 1998, 37, 550.

(32) Randall, G. C.; Doyle, P. S. Proc. Natl. Acad. Sci. U.S.A. 2005, 102, 10813

(33) Maier, B.; Rädler, J. O. Macromolecules 2000, 33, 7185.

(34) Ferree, S.; Blanch, H. W. Biophys. J. 2003, 85, 2539.

(35) Sevick, E. M.; Williams, D. R. M. Europhys. Lett. 2001, 56, 529

(36) Perkins, T. T.; Smith, D. E.; Chu, S. Science 1997, 276, 2016.

(37) de Gennes, P. G. Science 1997, 276, 1999.

(38) Viovy, J.-L. Science 1994, 264, 112.

(39) Doyle, P. S.; Shaqfeh, E. S. G.; McKinley, G. H.; Spiegelberg, S. H. J. Non-Newtonian Fluid Mech. 1998, 76, 79

(40) Bakajin, O. B.; Duke, T. A. J.; Chou, C. F.; Chan, S. S.; Austin, R. H.; Cox, E. C. Phys. Rev. Lett. 1998, 80, 2737.

(41) Randall, G. C. Single Molecule Analysis of DNA Electrophoresis in Microdevices. Ph.D. Thesis, Massachusetts Institute of Technology, 2006.

(42) Brochard-Wyart, F. Europhys. Lett. 1995, 30, 387

(43) Szabo, A.; Schulten, K.; Schulten, Z. J. Chem. Phys. 1980, 72, 4350.

(44) Doyle, P. S.; Bibette, J.; Bancaud, A.; Viovy, J.-L. Science 2002, 295, 2237.

(45) Ajdari, A.; Brochart-Wyart, F.; Gay, C.; de Gennes, P.-G.; Viovy, J.-L. J. Phys. II 1995, 5, 491

(46) Barron, A.; Soane, D.; Blanch, H. J. Chromatogr. 1993, 652, 2.

(47) Larson, R. G. The Structure and Rheology of Complex Fluids; Oxford University Press: Oxford, 1999.

(48) Tegenfeldt, J. O.; Bakajin, O.; Chou, C.-F.; Chan, S. S.; Austin, R.; Fann, W.; Liou, L.; Chan, E.; Duke, T.; Cox, E. C. Phys. Rev. Lett. 2001, 86, 1378

(49) Chan, E. Y.; Goncalves, N. M.; Haeusler, R. A.; Hatch, A. J.; Larson, J. W.; Maletta, A. M.; Yantz, G. R.; Carstea, E. D.; Fuchs, M.; Wang, G. G.; Gullans, S. R.; Gilmanshin, R. Genome Res. 2004, 14, 1137.

(50) Larson, J. W.; Yantz, G. R.; Charnas, R.; D’Antoni, C. M.; Gallo, M V.; Gillis, K. A.; Neely, L. A.; Phillips, K. M.; Wong, G. G.; Gullans, S. R.; Gilmanshin, R. Lab Chip 2006, 6, 1187.

(51) Randall, G.; Schultz, K.; Doyle, P. S. Lab Chip 2006, 6, 516.

(52) A persistent steeper postimpact slope of $x_{\text {com }}(t)$ was observed previously by others in a moderately dense magnetic bead obstacle field. ${ }^{25} \mathrm{We}$ speculate that their unexplainable persistent increase in slope was due to the peculiarities of that particular device, perhaps from acceleration of a stretched configuration in the field gradients near the surfaces of the many surrounding obstacles.

(53) Note the difference between $N$, the theoretical number of Kuhn lengths, and $N_{\mathrm{b}}$, the number of beads in the bead-spring model. $N=L / l_{\mathrm{k}}$ and $N_{\mathrm{b}}=x_{\mathrm{ex}} / l_{\mathrm{s}}$, where $l_{\mathrm{s}}$ is the average spring length for the nonuniform tension chain.

(54) The J model predicts an infinite trapping time for a symmetric hook with $x_{1}(0)=L / 2$. This makes sense since unhooking is driven by asymmetry. However, this singularity is integrable, and it can even be made finite by adding fluctuations.

MA061375T 